\title{
Il legato dei legami. Le sedi storiche dell'associazionismo italiano a Buenos Aires
}

\author{
Maria Pompeiana larossi \\ Cecilia Santacroce
}

Abstract

\begin{abstract}
II forte flusso di migrazione italiana che investì Buenos Aires a cavallo tra il XIX e il XX secolo coincise con la costruzione della nuova Capital federale. La maggior parte dei migranti vennero impiegati nei cantieri edili sia come maestranze che come tecnici, contribuendo così a dare un'impronta italianizzante alla città. Contrariamente a come accadde in altre città, essi non si concentrarono in quartieri etnicamente omogenei, ma s'insediarono diffusamente in tutta la città e, per ovviare alla dispersione della comunità, si istituirono numerose associazioni, arrivando a contarne già 75 solo nel 1909. Queste, di cui ad oggi 3 I sono ancora esistenti, attive e collocate nella sede originale, spesso occupano edifici propri e sono dotate di una riconoscibile ed autonoma identità architettonica, tanto da costituire un patrimonio costruito degno di essere studiato. L'analisi delle sedi, svolta attraverso un preliminare censimento con geolocalizzazione da remoto, integrato da sopralluoghi in situ ed infine dall'approfondimento di alcuni casi studio, hanno posto in luce come le caratteristiche distributive degli spazi, la composizione delle facciate e la collocazione all'interno del tessuto urbano bonaerense, riconducano la loro architettura entro un modello analogo a quello dell'architettura domestica alto-borghese, quasi ad affermarne il valore di casa collettiva di riferimento per il gruppo sociale che ne ha promosso la costruzione e che idealmente vi si riconosce.
\end{abstract}

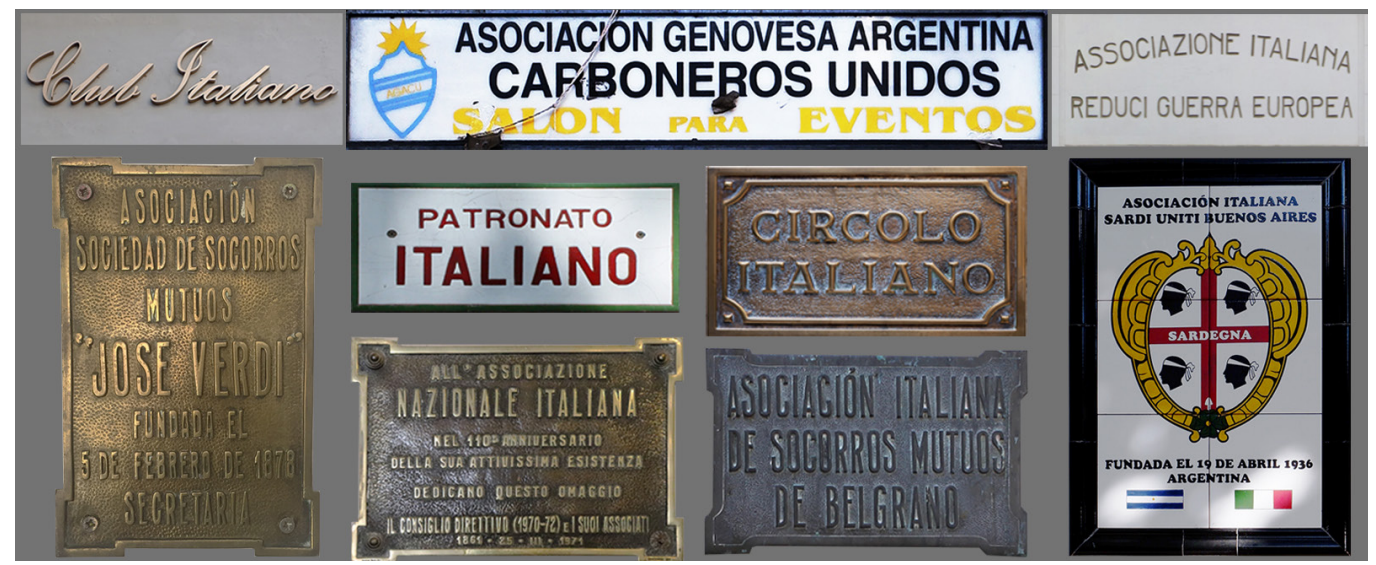




\section{L'eredità del fenomeno dell'associazionismo italiano a Buenos Aires}

Ogni fenomeno migratorio, quale che sia la ragione che lo ha indotto, stabilisce legami che tracciano una rotta, lungo la quale nel tempo sono destinate a viaggiare in ambedue le direzioni notizie, conoscenze, affetti e visioni del mondo, determinando così una relazione biunivoca tra due luoghi, i cui caratteri confluiscono sinergicamente a creare fenomeni culturali ed insediativi di grande interesse.

La lettura dei rapporti tra Italia e Argentina appare di particolare rilevanza, in primo luogo per l'entità dei flussi migratori, considerato che, solo tra il I880 e il 1930, sbarcarono a Buenos Aires 2.200.000 Italiani. Dopo una permanenza obbligatoria di cinque giorni nella gigantesca struttura di accoglienza de l'Hotel de Inmigrantes, (fig. I) quelli fra essi che provenivano dai contesti rurali, venivano indirizzati verso le regioni meridionali, dove era iniziata la grande epopea della conquista delle terre patagoniche. Ma la gran parte di essi finiva per fermarsi a Buenos Aires [I], trovando lavoro nel porto e, soprattutto, nei numerosi cantieri edilizi aperti in tutta la città, che dal 1880 aveva avviato il processo di trasformazione del suo volto in quello di Capital dello Stato federale, conferendo al suo volto una comune matrice costruttiva e stilistica.

Sebbene i modelli culturali ed economici adottati dall'intelligencija argentina fossero rispettivamente quello francese e quello inglese, l'architettura reca un'impronta prettamente Italianizante, dovuta al fatto che l'edilizia era quasi totalmente nelle mani de los Tanos (gli Italiani), impegnati non solo come muratori, ma anche come architetti, ingegneri, decoratori e artigiani. Si trattava di maestranze e tecnici portatori di una formazione appresa nel paese d'origine, accentuata dal fatto che taluni materiali e, talvolta, perfino manufatti completi (marmi e ceramiche, ma anche ringhiere e inferriate in ferro battuto o vetrate decorative) venivano fatti arrivare via mare direttamente dall'Italia.
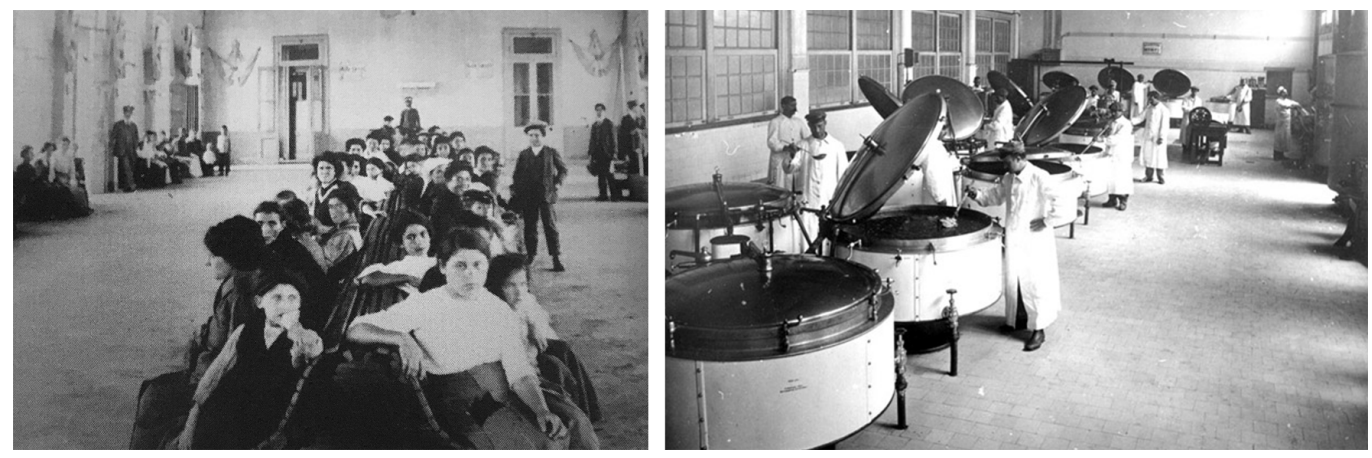

Il know-how edilizio era dunque saldamente detenuto dalla comunità italiana, numericamente consistente e, nel suo insieme, in rapida ascesa economica e sociale, favorita anche dalle energiche politiche d'integrazione culturale adottate dallo Stato. Fra esse, in primo luogo la Ley de Educación común del I 884 - che istituiva dell'obbligo scolastico laico, universale e gratuito fino a I 4 anni - il cui piano di attuazione del I 899 (il PPE-Plan de Edificación Escolar) in pochi anni dotò la Capital e l'intero territorio nazionale di una capillare rete di edifici per l'istruzione primaria [2] [D'Amia, larossi 20 I8], alla cui progettazione presiedette l'italiano Carlos Morra, ancora una volta con il contributo di maestranze, tecnici e progettisti italiani. Grazie a queste politiche di accoglienza, a Buenos Aires gli emigrati non si concentravano in quartieri etnicamente omogenei come Little Italy a New York, ma s'insediavano diffusamente in tutta la città, con l'unica eccezione del quartiere de La Boca, dove c'era una consistente comunità di Genovesi, impiegati come scaricatori, barcaioli e piloti presso il porto fluviale allo sbocco del Riachuelo [3]. 
Il bisogno di aggregazione in comunità, in cui la comune provenienza italica costituisse un fattore di radicamento in un paese estraneo e in una dimensione urbana assai vasta già allora - e che ancor più gigantesca doveva apparire ai migranti dalle province italiane - a partire dalla metà del XIX secolo diede impulso all'istituzione di numerosissime associazioni di mutuo soccorso.

Inizialmente organizzati su base regionale o di scopo (ad esempio, per fornire assistenza medica su base solidale), circoli e sodalizi divennero presto per la comunità italo-argentina i fulcri della vita associata. Successivamente, con l'aumento dei flussi migratori, comparvero anche società con altri scopi - politico, culturale, assistenziale, sportivo, ricreativo o d'istruzione - come anche forme aggregative riferite al barrio bonaerense di ubicazione.

Alcune di tali associazioni ebbero vita breve, altre invece prosperarono notevolmente, grazie al costante incremento del numero degli iscritti, che permise e rese necessaria la realizzazione di edifici appositamente destinati ad ospitarle.

Essi hanno rappresentato - e spesso tutt'ora rappresentano - un punto di riferimento fondamentale per gli emigranti italiani dispersi nella metropoli, rispondendo non solo al bisogno di socializzazione, ma garantendo anche il soddisfacimento di esigenze primarie, come l'assistenza sanitaria o la formazione professionale.

Molte delle associazioni nate a cavallo tra il $\mathrm{XIX}$ e il $\mathrm{XX}$ secolo sono, infatti, ancor oggi presenti nei diversi barrios della città e gli edifici che le ospitano continuano ad intessere forti reti di aggregazione socioculturale entro una realtà demograficamente eterogenea e mutevole a causa dell'afflusso tutt'ora costante di nuovi immigrati (ad esempio, di recente, dal Venezuela).

Si tratta di un patrimonio che spesso versa in precario stato di conservazione a causa delle deboli politiche di tutela vigenti nel Paese e reso fragile dalla sua stessa condizione di diffusione entro il tessuto della metropoli, ma indubbiamente esemplare e meritevole di conoscenza e valorizzazione, sia come strumento d'integrazione sociale e culturale e sia da un punto di vista strettamente architettonico (fig. 2). (M.P.I.).

Fig. 2. Le associazioAires rappresentano una relà importante strumento di coesione sociale (foto degli autori).
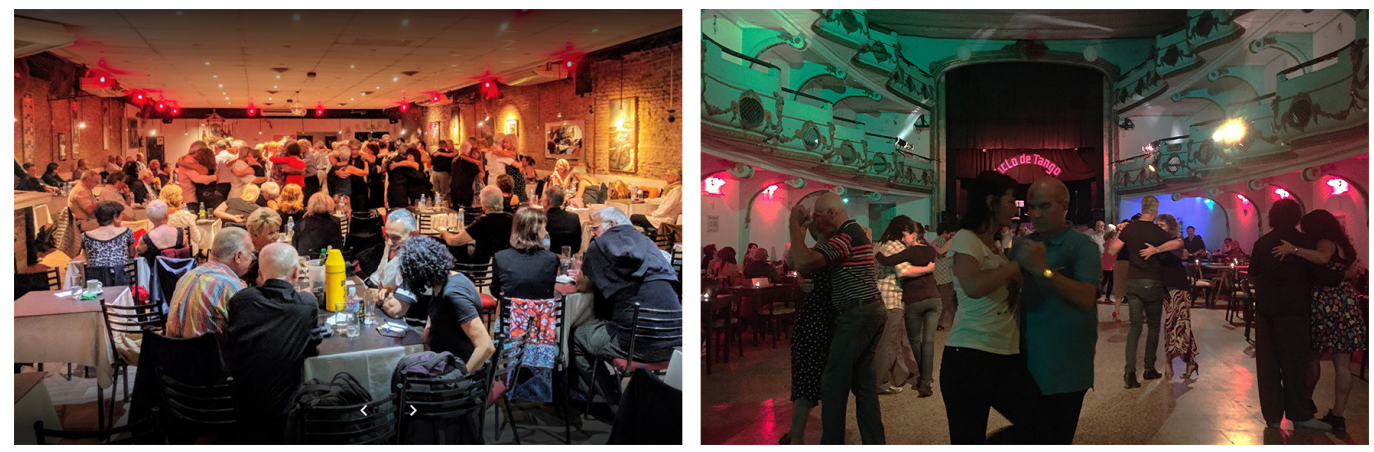

\section{Fonti e metodologie d'indagine}

II numero di studi sul fenomeno della migrazione italiana in Argentina e sul ruolo sociale ed economico svoltovi dall'associazionismo [4] è veramente cospicuo [5].

Invece - se si escludono i casi più eclatanti e con valenza monumentale, come, ad esempio, l'edificio dell'Unione e Benevolenza [6] risulta assai poco indagato l'insieme degli edifici destinati a sedi delle associazioni, sebbene essi compongano un lascito cospicuo e di notevole interesse.

Al fine di comprendere il ruolo svolto da tali edifici nella fase in cui Buenos Aires andava delineando il proprio volto di Capital, si è ritenuto di circoscrivere l'indagine al periodo compreso tra il 1880 e il 1910, che coincide anche con il momento di massima intensità dei flussi migratori. 
Fig. 3. A sinistra, estratto del database: in verde scuro i casi studio rilevati, in verde chime gli edifi in verde chiaro gil edifici chiaro quelli chi, in giallo chiaro quelli che hanno subito un cambio di destinazione d'uso, in rosso gli edifici demoliti. A destra, esempio di scheda di censimento (elaborazione degli autori).
Lo studio ha quindi preso le mosse dai primi elenchi ufficiali dei sodalizi italiani all'estero riconosciuti dal Ministero degli Esteri [7] confrontati con l'elenco delle associazioni indicate come ancora attive a Buenos Aires dal sito del medesimo ministero [8].

La lettura incrociata di tali dati è confluita in un database con relativa geolocalizzazione da remoto di 9 I edifici, in base a cui è stato possibile procedere in situ [9] alla verifica dei dati precedentemente acquisiti, alla schedatura descrittiva degli edifici fisicamente individuati e al rilievo dei casi-studio, selezionati per la loro paradigmaticità (fig. 3).

La dispersione del patrimonio indagato entro una superficie urbana assai vasta, ha suggerito di adottare procedure mensorie di tipo speditivo, basate sull'integrazione del rilievo diretto con la fotografia e la fotogrammetria 3D, nonché supportate da ricerche documentali, condotte negli archivi storici, laddove presenti, delle singole associazioni.

È stato così possibile predisporre dei materiali descrittivi, che hanno rappresentato la base per successive elaborazioni ed analisi, sviluppate in riferimento alla comprensione sia dei caratteri architettonici peculiari di ciascun edificio e sia del rapporto stabilito di volta in volta con il tessuto urbano circostante e con la struttura complessiva della città.

Con riferimento alle metodologie analitiche, pare opportuno evidenziare che, in ragione sia della dispersione del patrimonio indagato e sia dei caratteri specifici della città di Buenos Aires, è parso di particolare interesse l'approfondimento dei caratteri architettonici delle facciate.

A Buenos Aires, infatti, la scarsità di vincoli sia nel processo edificatorio della maglia isotropa degli isolati (derivante dall'estensione della griglia fondazionale fissata nel XVI secolo dalle Leyes de India) e sia in quello di sostituzione edilizia dei secoli successivi [Gorelik 1998] ha prodotto un tessuto edilizio alquanto variegato sotto il profilo tipologico e linguistico. In ragione di questa sua peculiarità, appare fondamentale appprofondire lo studio dei fronti edilizi, poiché il carattere architettonico precipuo della città risiede proprio nella tensione fra la monotonia illimitata del reticolo delle cuadras e l'incredibile ricchezza semantica caratterizzante le facciate degli edifici. (M.P.I.).

\begin{tabular}{|c|c|c|c|c|c|c|}
\hline Denominacion & Domilitio & Datat af it ondaraione & Teleteno & Emal & Notes & Date \\
\hline 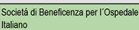 & Gascón 450 , CABA & 1853 & 49584462 & & 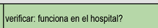 & 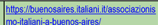 \\
\hline 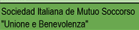 & Tre Gereat unan D. Peror 1352 & 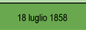 & & & & 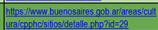 \\
\hline 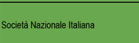 & 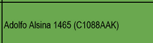 & $25 \operatorname{marc} 1889$ & & 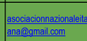 & 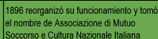 & 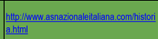 \\
\hline Recuvid dille parie Batragle & 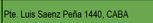 & nementeren 1899 & 43044476 & & 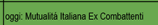 & \\
\hline croob tallem & 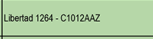 & 1873 & & 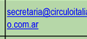 & 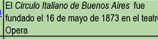 & 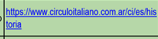 \\
\hline Inimene Opesai thatari & Sammeno 1374 & Geenao 1874 & & & 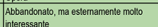 & 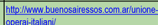 \\
\hline 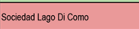 & 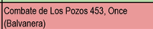 & 1 mero 1876 & $011+42924065$ & & 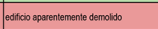 & \\
\hline Colonil latarna & Pararat 555 & 8 saplit 1877 & & & 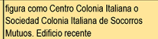 & \\
\hline Sthela chlatian & Rvedetra 613 & 1878 & & & & \\
\hline Satia e Lavoro & Cnle 1567 & 17 tebtrab 1878 & & & 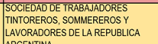 & \\
\hline thisuntas & 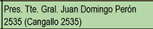 & 5 manos 1878 & & & & \\
\hline Marghentia o S Swoo & Carbs Cavo os 54 & $1900 \mathrm{ma} 01879$ & & & & \\
\hline 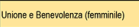 & Covallos 78 & 27 lysto 1079 & & & & \\
\hline bian & 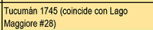 & 18 gennein 1883 & & & 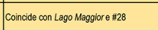 & \\
\hline Somonelata & 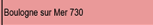 & 2 2ugplo 1883 & & & 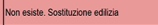 & \\
\hline Crovo Sannition & & 8 dicembre 1883 & & & & \\
\hline exsatenomber & Alsmo 2325 & 14 stementre 1824 & & & 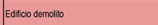 & \\
\hline Unioro Hardicanale & Sanzz Peñia 1046 & 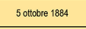 & & & & \\
\hline Camera It alana si Commecicio & 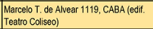 & 1 dicentrer 1894 & & & 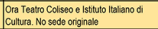 & \\
\hline Societá Cucche Cemerenei & & ${ }_{1884}$ & & & & \\
\hline 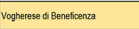 & 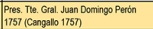 & 59y40018887 & & & & \\
\hline
\end{tabular}

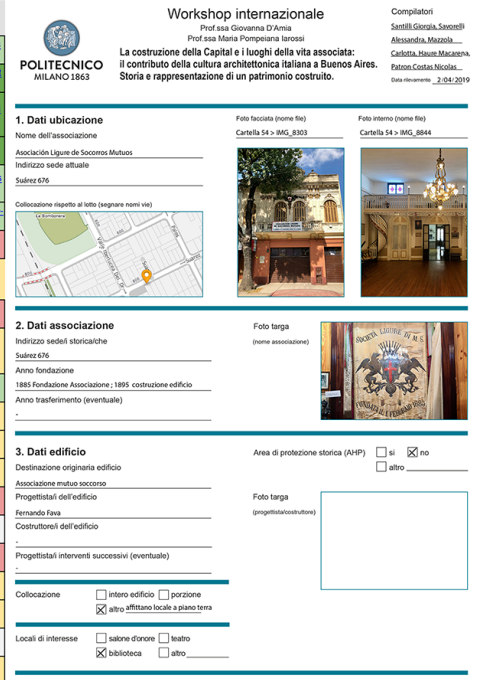

\section{Censimento, mappatura, schedatura e rilievo delle sedi di associazioni italiane a Buenos Aires}

Dal primo censimento del Ministero Italiano degli Affari Esteri, risulta che nel 1909, nella sola capitale erano attivi ben 75 sodalizi, fondati a partire dalla metà del XIX secolo e di cui 13 con un numero di iscritti superiore al migliaio [I0]. I successivi diversi censimenti pubblicati sul sito del Ministero Affari Esteri [ I I ] consentono tuttavia di desumere come questo numero nel tempo sia variato, per effetto della chiusura o della fusione di alcune associazioni, 
Fig. 4. Localizzazione all'interno dei quartier della CABA degli edifici delle sedi delle associazioni italiane esistenti e demoliti (elaborazione grafica deg autori). così come della nascita di nuovi sodalizi; per cui, nel 20 I 8, risultavano 9| sedi di associazioni italiane ricadenti nel territorio della CABA-Comunidad Autónoma de Buenos Aires.

Di queste, dall'indagine in loco, 23 associazioni sono risultate sconosciute all'indirizzo riportato negli elenchi ministeriali; è stato perciò materialmente possibile identificare le sedi di solo 68 di esse, fra le quali ne sono state individuate 31 ancora attive e meritevoli di successivo approfondimento, in quanto dotate di una sede propria e coincidente con un intero edificio, realizzato appositamente per ospitarle. Tale scelta ha quindi escluso dall'ambito di approfondimento tutti i casi di sedi di associazione corrispondenti a porzioni di edifici non dotate di una loro riconoscibile ed autonoma identità architettonica.

Gli edifici così selezionati sono stati pertanto oggetto di ulteriore sopralluogo, durante il quale è compilata per ciascuno di essi una scheda, completa di tutti i dati identificativi sia del sodalizio e sia della sede, con relativo stato di conservazione e utilizzo, corredata dall'indicazione del progettista e dalla descrizione dei caratteri architettonici, tipologici, materici e stilistici dell'edificio, con relativa documentazione fotografica e grafica redatta in loco. In tale occasione si è altresì provveduto all'acquisizione dagli archivi delle associazioni di eventuale

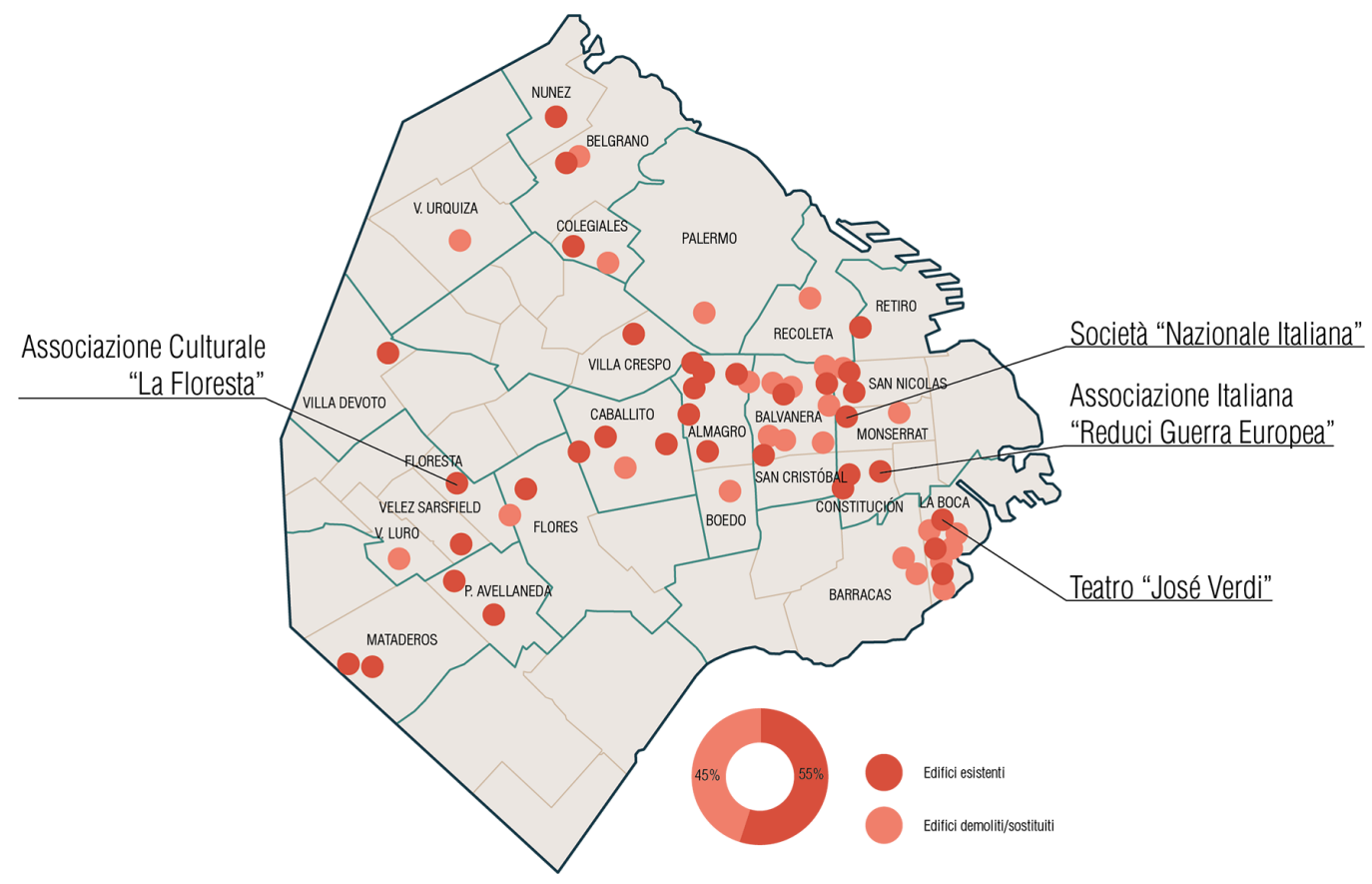

materiale storico rinvenuto e ritenuto d'interesse ai fini dell'indagine, con particolare attenzione alla documentazione relativa ad eventuali modifiche subite dall'edificio nel corso del tempo.

Sulla base di tale lavoro, nonché di considerazioni relative alla effettiva ed agevole accessibilità è stato infine possibile selezionare quattro casi studio:

- Teatro "José Verdi" (La Boca);

- Associazione Italiana "Reduci Guerra Europea" (Costitución);

- Associazione Culturale "La Floresta" (Floresta);

- Società "Nazionale Italiana" (Monserrat).

Tali edifici - selezionati in modo da garantire varietà di datazione, autore, ubicazione nella città e collocazione nell'isolato - sono stati oggetto di analisi in dettaglio, condotta sulla scorta del rilievo diretto condotto ad integrazione dei modelli digitali delle facciate e dei più significativi spazi interni (come atri e saloni d'onore) [I2] (fig. 4). (M.P.I.). 


\section{Caratteri peculiari degli edifici sedi di associazioni italiane a Buenos Aires}

Con riferimento al lavoro di mappatura e censimento, eseguito da remoto, è stato possibile osservare e trovare conferma della distribuzione disseminata nei diversi barrios della città, indicando tuttavia una più marcata concentrazione nei quartieri di Balvanera, La Boca e Almagro.

Si è inoltre riscontrato che essi, generalmente, occupano un lotto singolo, sviluppato nella profondità dell'isolato e collocato in posizione baricentrica rispetto al fronte urbano della cuadra anche se non mancano casi in cui essi occupano posizioni d'angolo (fig. 5). La sede dell'associazione, in tal modo, costituisce parte integrante della cortina stradale formata dal tessuto residenziale contiguo.

Allinterno della monotona compagine urbana e rispetto al tessuto edificato circostante, l'edificio dell'associazione si distingue solo per l'architettura della sua facciata posta a fare da diaframma tra lo spazio urbano pubblico della strada e lo spazio privato interno, quasi a voler comunicare visivamente il ruolo di radicamento socioculturale dell'istituzione ospitata, segnalata dalla presenza di una targa metallica o di un'insegna, sempre ben visibile e di significative dimensioni (fig. 6).

La facciata, nella maggior parte dei casi esaminati, è risultata simmetrica, scandita da cornici marcapiano e articolata con l'uso gerarchizzato degli ordini classici, concludendosi nel coronamento, spesso costituito da frontone o cornicione con muro d'attico ed elementi scultorei (figg. 7, 8).

Questi fronti presentano infatti caratteri edilizi non disomogenei rispetto al tessuto residenziale storico della cortina muraria circostante, caratterizzata da sviluppo altimetrico di uno/due piani fuori terra, su basamento modestamente rialzato rispetto alla quota stradale. Solo alcuni elementi linguistici e decorativi - come l'insegna, il cornicione di coronamento monumentale e la presenza di un'apertura al centro della facciata, talvolta sottolineata da balcone centrale, ma sempre dotata di asta porta-bandiera - ne consentono la riconoscibilità rispetto al tessuto abitativo, quasi a sottolineare il carattere di casa comune per la collettività che in tali edifici è destinata a riconoscersi.

L'articolazione distributiva dello spazio interno ha come fulcro ed elemento di prestigio il salone d'onore, spesso a doppia altezza, preceduto da un ampio vestibolo d'ingresso con scala di rappresentanza (fig. 9).

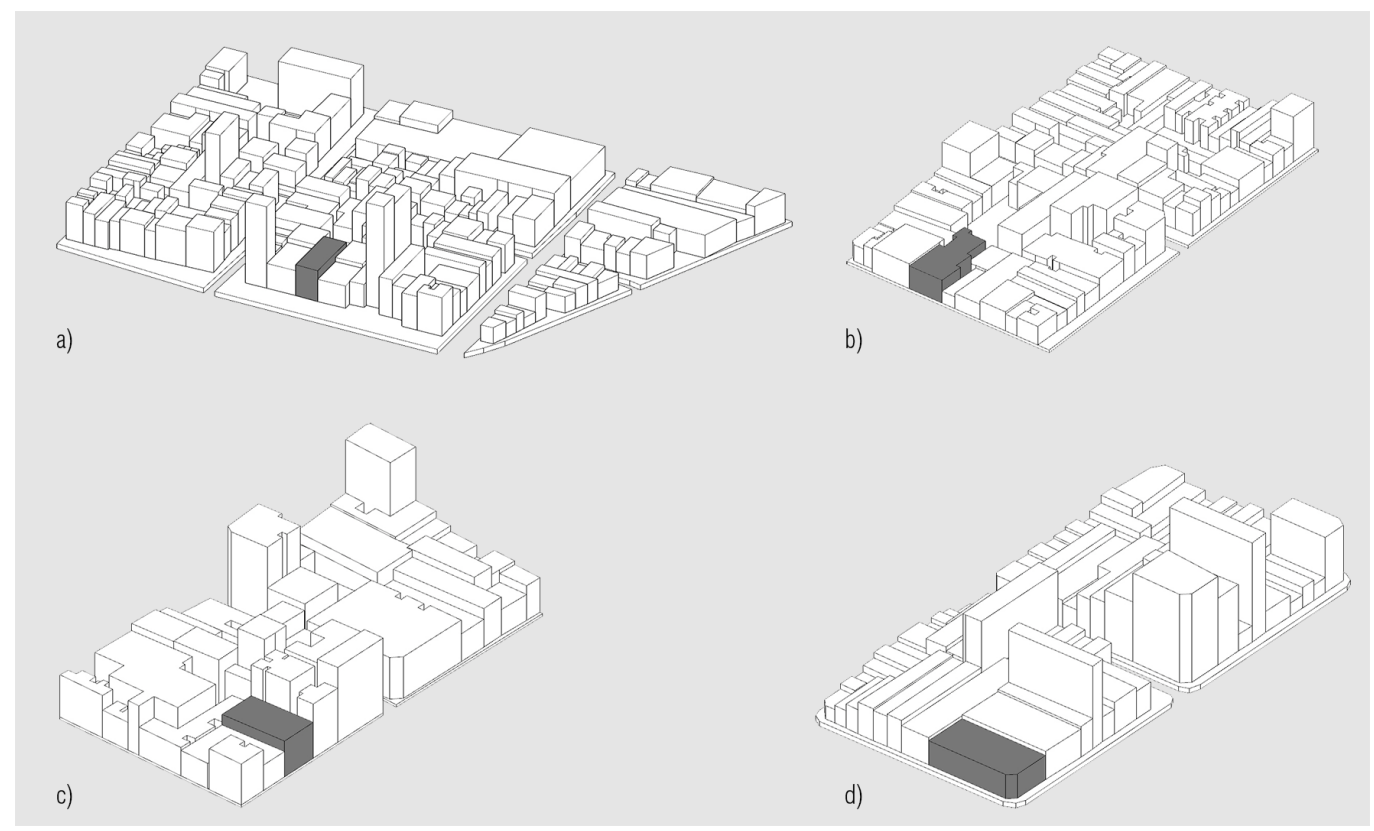


Fig. 6. Insegne e targhe di alcune associazioni

ancora attive (foto degli autori).
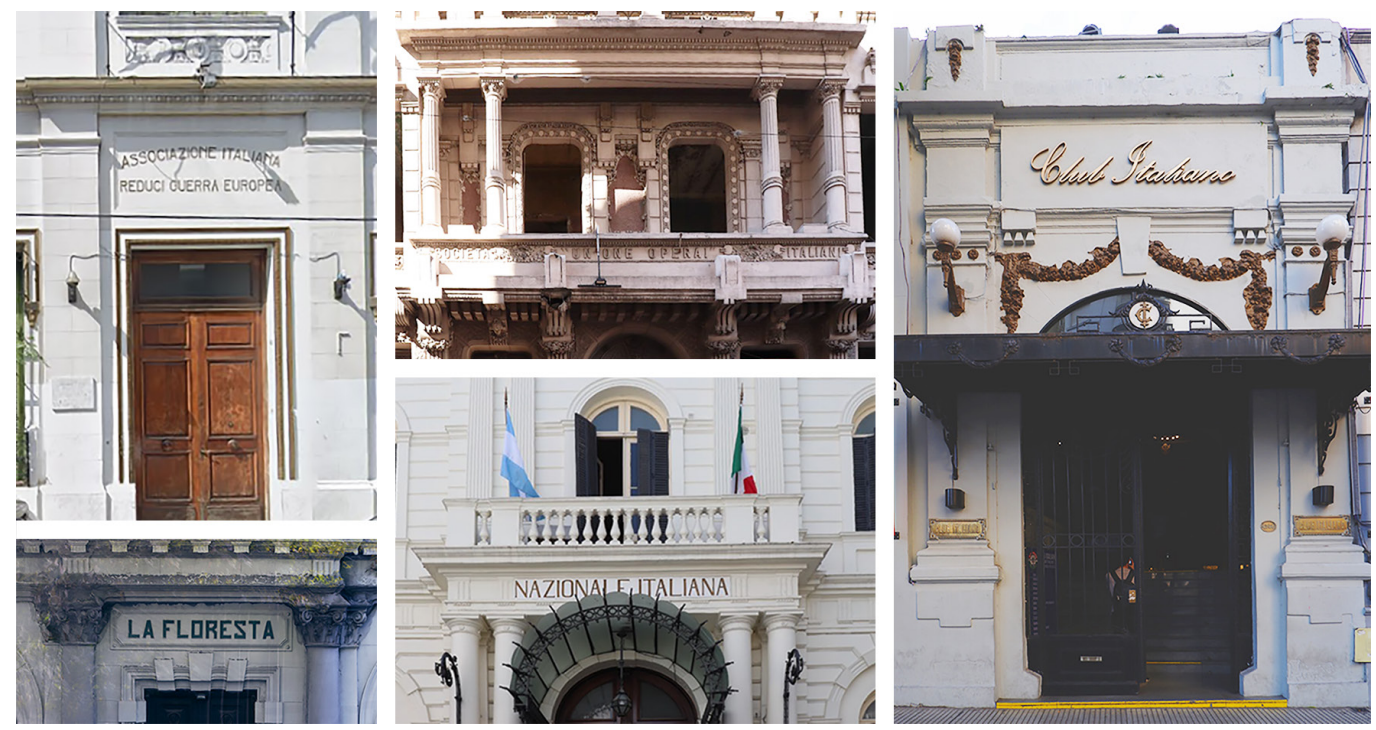

Fig. 7. Elaborazione con Agistoft Photoscan delle Agisto thotoscan "José facciate del Teatro "José ver " dell Associazione Italiana "Reduci Guerra
Europea" (elaborazione Europea" (elaborazio
grafica degli autori).
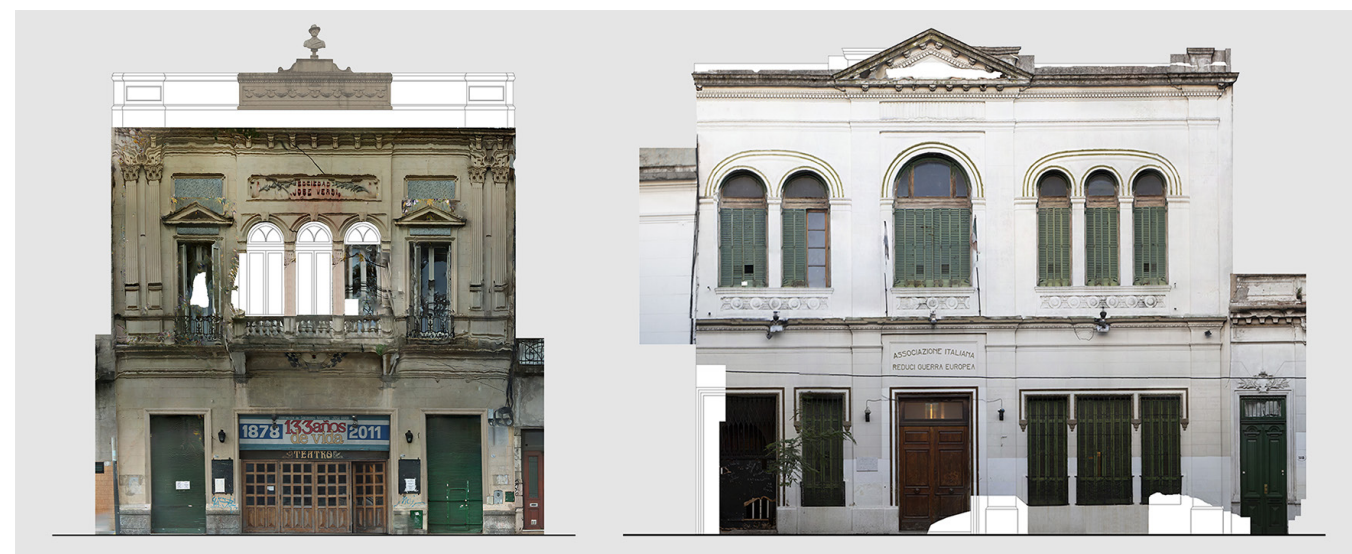

Fig. 8. Elaborazione con Agisoft Photoscan delle facciate dell'Associazione Culturale "La Floresta" e della Società "Nazionale Italiana" (elaborazione grafica degli autori).

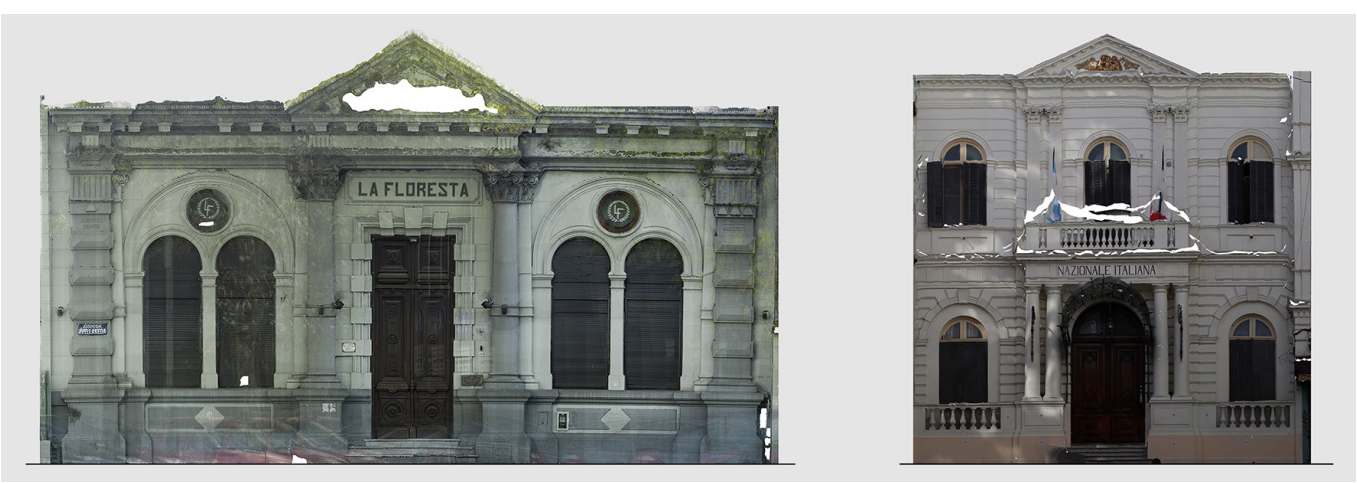


Fig. 9. Elaborazione con Agisoft Photoscan e fodell'Associazione Italiana "José Verdi" (elaborazione grafica degli autori).
Nella maggior parte dei casi il salon si configura come un vero e proprio teatro, dotato di una o più gallerie e di un palcoscenico con tutti i suoi locali accessori.

Alcuni di questi spazi sono tutt'ora in funzione; ospitano spettacoli teatrali o musicali e vengono utilizzati il più delle volte come scuole di tango e milongas, le sale da ballo, diffuse in tutta l'area rioplatense, destinate specificatamente al tango.
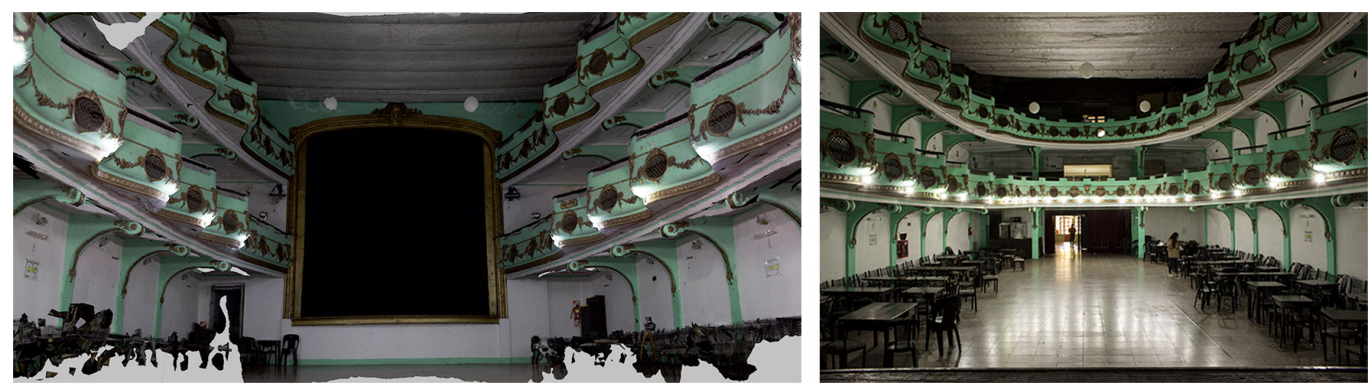

Di particolare interesse è risultato il salone della Società di Mutuo Soccorso José Verdi, che risulta a tutti gli effetti una sala teatrale (fig. I0). È infatti munita di un vero e proprio palcoscenico, chiuso da arco scenico, che si apre su un'ampia sala, circondata da due ordini di balconate e coperta da un tetto metallico apribile. Purtroppo, le balconate sono attualmente inagibili perché pericolanti e il meccanismo di apertura del tetto non è più funzionante. Ciò nonostante, l'associazione continua a rappresentare non solo un luogo di riferimento per gli abitanti del quartiere de La Boca in cui si trova, ma anche un polo attrattivo per i fautori del tango nella sua forma originaria [13], anche se residenti in altri quartieri della città.
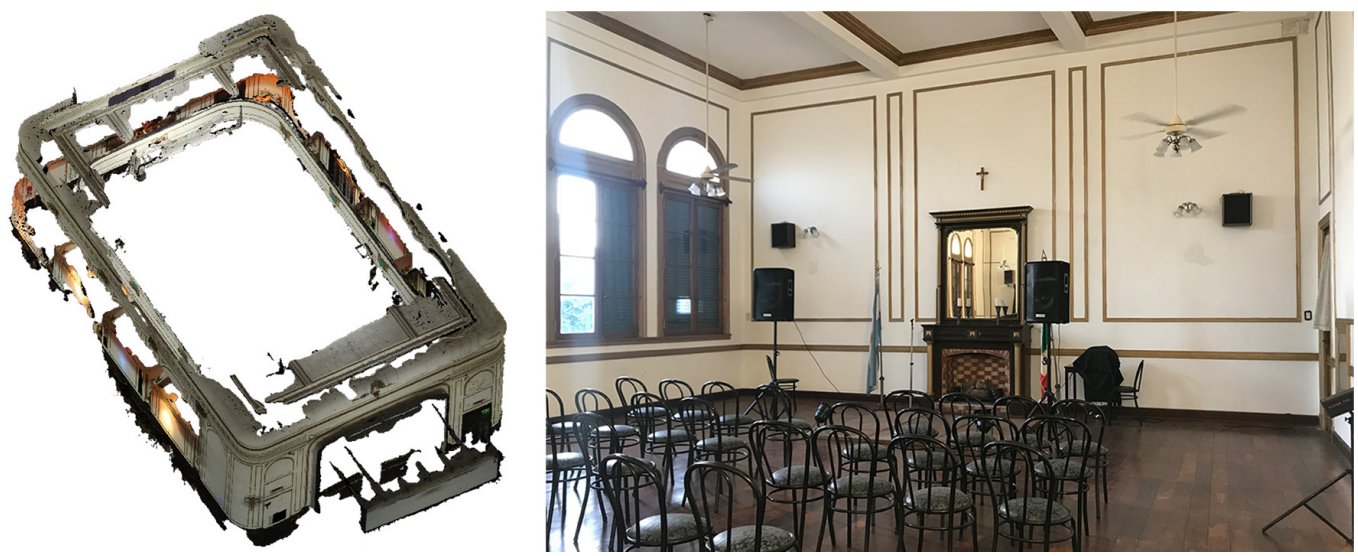

Attorno al salone ed ai piani superiori si organizzano e gli ambienti collaterali, differenziati a seconda delle attività e dei servizi offerti da ciascuna associazione. Sono infatti presenti, uffici, aule per l'istruzione, biblioteche, sale riunioni, studi medici, piccoli musei, bar e sale per lo sport (fig. I I).

L'indagine svolta sulle sedi delle associazioni italiane a Buenos Aires ha mostrato come le caratteristiche distributive riscontrate e la collocazione nella compagine dell'isolato e della città, riconducano l'architettura di tali edifici entro la matrice dell'architettura domestica alto-borghese, mentre la composizione delle facciate e la presenza di taluni elementi decorativi attribuiscono loro una connotazione monumentale, quasi ad affermarne, da una parte, il valore di casa e, dall'altro, la natura collettiva, di riferimento per il gruppo sociale che ne ha promosso la costruzione e che idealmente vi si riconosce. (C. S.). 
Fig. II. Organizzazione degli ambienti laterali e degli spazi di servizio al salone d'onore nelle associazioni "Reduci Guerra Europea" (a) "Nazionale Italiana" (b) e nel Teatro "José Verd" (c); (elaborazione grafica degli autori).

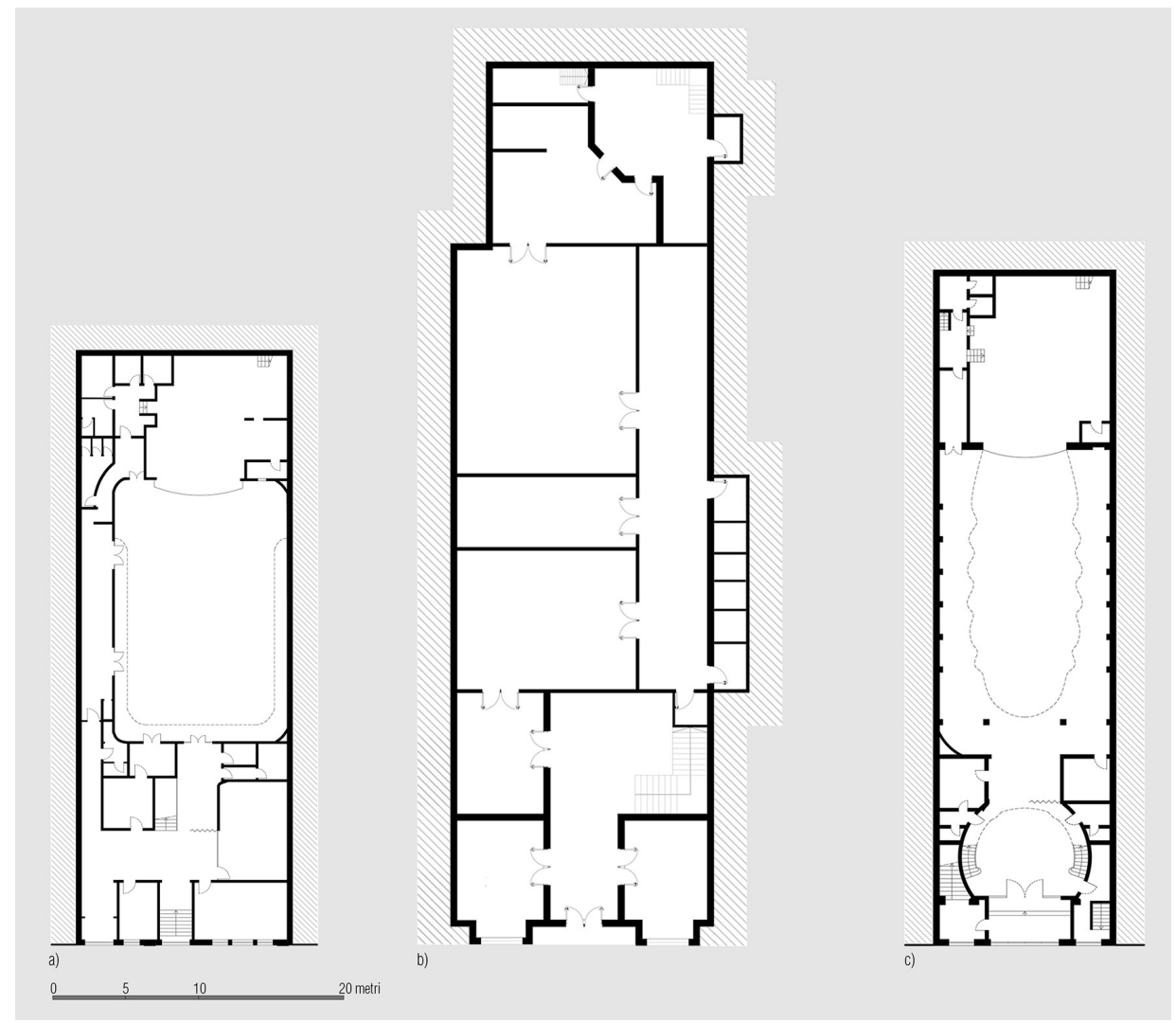

Note

[I] |l primo censimento nazionale argentino ( 869 ) rileva che "il 59\% di tutti gli italiani in Argentina si stabiliva a Buenos Aires": [Ministero degli Affari Esteri 2003, p. 4]

[2] Brandariz 1998.

[3] Barzini 1902, pp. 19-21; larossi 2017, pp. 14-26.

[4] II ruolo di legante per la comunità italo-argentina svolto dall'associazionismo era già stato ampiamente riconosciuto da L. Barzini fin dal 1901, durante la sua permanenza a Buenos Aires come inviato speciale del Corriere della Sera: Barzini 1902 pp. $166-172$.

[5] Per una ricostruzione storica dello sviluppo dell'associazionismo a Buenos Aires, vedasi: Baily 1999, pp. 174 - 176.

[6] Per una trattazione delle vicende storiche dell'Unione e Benevolenza, la più antica associazione italiana a Buenos Aires (fondata nel 1855), nonché per una descrizione del vasto e complesso edificio che ne costituisce la sede, ospitandovi un teatro, saloni, uffici, aule e sale riunioni, vedasi: Cibotti 1986.

[7] Zuccarini 1909, pp. I52-156.

[8] Si veda: <http://www.esteri.it/mae/doc_osservatorio/gli_italiani_in_argentina.pdf>.

[9] Le operazioni di censimento e rilevamento sul campo sono state avviate all'interno del workshop "Storia e Rappresentazione di un patrimonio costruito" svolto nell'aprile 2019 a Buenos Aires in partnership tra Scuola AUIC- Architettura, Urbanistica e Ingegneria delle costruzioni del Politecnico di Milano e dalla Facultad de Arquitectura y urbanismo della Universidad de Belgrano a Buenos Aires. I materiali prodotti in situ sono stati poi oggetto in Italia di un cospicuo lavoro di elaborazione e approfondimento.

[10] Zuccarini 1909, pp. 152-156.

[I I] Si veda: <https://www.esteri.it/mae/doc_osservatorio/rapporto_italiani_argentina_logo.pdf>.

[12] Ciascun modello 3D è stato realizzato tramite fotomodellazione digitale, con l'elaborazione d'immagini acquisite con fotocamera digitale CANON EOS I00D e rielaborate col software Agisoft Photoscan. Per essere efficaci ai fini del rilievo, tali immagini sono state acquisite con modalità speditiva utilizzando una stessa ottica per tutto il set di lavoro e predisponendo che ciascun fotogramma sia sovrapposto di almeno un 30\% rispetto a quelli adiacenti, al fine di garantire una corretta coincidenza dei punti omologhi.

[I 3] Si rammenta che il tango nel 2009 è stato dichiarato dall'Unesco appartenente al Patrimonio Immateriale dell'Umanità. 


\section{Riferimenti bibliografici}

Baily Samuel L. (1999). Immigrants in the Lands of Promise. Italians in Buenos Aires and New York City, 1870-1914. Ithaca and London: Cornell University Press.

Barzini Luigi (1902). L'Argentina vista com'è. Milano:Tipografia del Corriere della Sera.

Brandariz Gustavo (1998). La arquitectura escolar de inspiración sarmentina. Buenos Aires: FADU-UBA.

Capocaccia Fabio, Pittarello Liliana, Rosso Del Brenna Giovanna (2016). Storie di emigrazione: architetti e costruttori italiani in America Latina. Genova: Stefano Termanini Editore.

Cibotti Ema (1986). Mutualismo y política en un estudio de caso. La sociedad 'Unione e Benevolenza' en Buenos Aires entre I 858 y | 865. In Devoto Fernando J. e Rosoli Gianfranco. L'Italia nella società argentina. Roma: Centro Studi Emigrazione, pp. $24 \mid-265$

Gutiérrez, Ramón (2004). Los italianos en la arquitectura argentina. Aproximaciones històricas. In Boletín CEDODAL: Italianos en la Arquitectura Argentina, gennaio.

larossi M. Pompeiana, Santacroce Cecilia (2019). The Schools as Heritage and a Tool for Political and Cultural Integration. The Buildings of the Plan de Edificación Escolar in Buenos Aires. In Della Torre Stefano, Bocciarelli Massimiliano, Daglio Laura, et al. Buildings for Education. Berlin: SpringerVerlag, pp. 73-85.

larossi M. Pompeiana (20 I 7). Laboratorio La Boca. Tracce d'Italia a Buenos Aires. Firenze: Altralinea Edizioni.

Zuccarini Emilio (1909). Il lavoro degli Italiani nella Repubblica argentina: dal 15 I 6 al 19 I0: studi, leggende e ricerche. Buenos Aires: Oficine gráfiche de la Compañia General de Fósforos.

Ministero degli Affari Esteri. Osservatorio sul Lavoro e sulla Formazione degli Italiani all'Estero. Gli Italiani in Argentina. In: <http:// www.esteri.it/mae/doc_osservatorio/gli_italiani_in_argentina.pdf $>$; $<$ https://www.esteri.it/mae/doc_osservatorio/rapporto_ italiani_argentina_logo.pdf>

Portal de la Comunidad Italiana en Argentina <http://www.feditalia.org.ar/>.

Autori

Maria Pompeiana larossi, Politecnico di Milano, mariapompeiana.iarossi@polimi.it

Cecilia Santacroce, Politecnico di Milano, cecilia.santacroce@polimi.it

Per citare questo capitolo: larossi Maria Pompeiana, Santacroce Cecilia (2020). Il legato dei legami. Le sedi storiche dell'associazionismo italiano a Buenos Aires/Legacy of links. The historical headquarters of Italian associationism in Buenos Aires. In Arena A., Arena M., Brandolino R. G., Colistra D., Ginex G., Mediati D., Nucifora S., Raffa P. (a cura di). Connettere. Un disegno per annodare e tessere. Atti del $42^{\circ}$ Convegno Internazionale de Docenti delle Discipline della Rappresentazione/Connecting. Drawing for weaving relationships. Proceedings of the 42th International Conference of Representation Disciplines Teachers. Milano: FrancoAngeli, pp. 2292-231. 


\title{
Legacy of Links. The Historical Headquarters of Italian Associationism in Buenos Aires
}

\author{
Maria Pompeiana larossi \\ Cecilia Santacroce
}

\section{Abstract}

The massive Italian migratory flux which hit Buenos Aires between the XIX and the XX centuries corresponded with the construction of the new federal Capital. Most of migrates were hired on construction sites, both as workers and as technicians, contributing in this way to give an Italianized sign to the city. Contrary to how it happened in other cities, the migrates didn't concentrated themselves in ethnically homogeneous neighbourhoods, but they took up residence all over the city, so that to solve the community's dispersion a huge number of associations were established, arriving to count 75 only in 1909. These associations, of which $3 \mathrm{I}$ are already existing, active and situated in the original site, are often located in their own buildings and they have a recognizable and autonomous architectural identity, so that they can be considered like a built heritage worthy of being studied. The sites' analysis, made through a preliminary census from digital database, integrated to survey in situ and then the beep analysis of some study cases taken as a model, highlighted how the distribution of inner spaces, the facades composition and their position into the Buenos Aires' urban pattern, connect their architecture to an upper middle class housing model, as they want to confirm their value of common houses for those social group which promoted their building and in which it recognises itself.

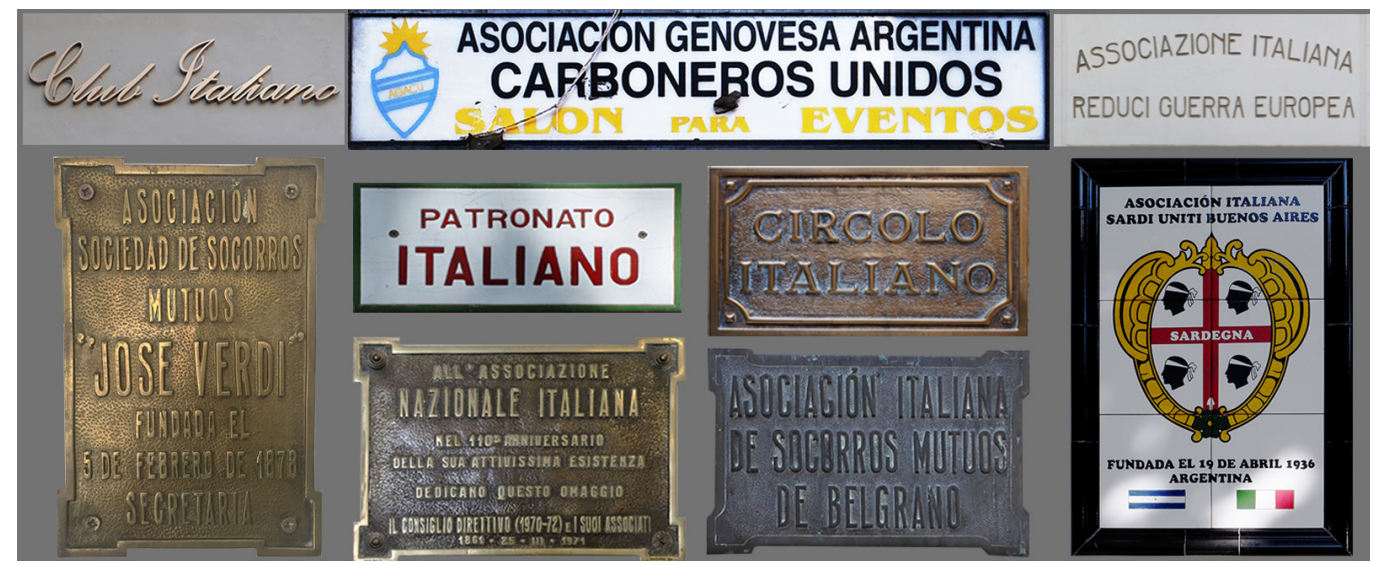




\section{The heredity of the Italian associationism phenomenon in Buenos Aires}

Every migration phenomenon, whatever it is the reason that provokes it, sets up bonds which draw a route along which news, knowledge, affections, visions of the world travel in each direction and in time. This establishes a bijective relationship between two places, which characters merge creating cultural and settlement phenomena of great interest.

The reading of the relationship between Italy and Argentina seems to be of particular interest, at first because of the importance of the migratory fluxes, in consideration that between I 880 and 1930, 2.200.000 of Italians arrived in Buenos Aires. After an obligatory permanence of five days in the big reception facility of Hotel de Inmigrantes (fig. I), the people that came from rural context were addressed to the southern regions, where the conquest of the Patagonian lands had begun. But most of the migrants stopped in Buenos Aires [I], finding job at the harbour and, especially, at the numerous construction sites opened all over the city, which since 1880 had begun the process of its transformation into the Capital of the Federal State, giving to itself a new constructive and stylistic matrix.

Even though the cultural and economic models used by the Argentine intelligencija were respectively the French and the English ones, the architecture had an Italianizante mark, because the construction industry was in the hands of los Tanos (the Italians), whom were hired not only like bricklayers, but also like architects, engineers, decorators and artisans. They were workers and technicians that brought an education learned in their native country, emphasized by the reason that some materials and, sometimes, some completed products (marble, ceramic, but also wrought iron railings and grating or decorative glass windows) came by sea directly from Italy.
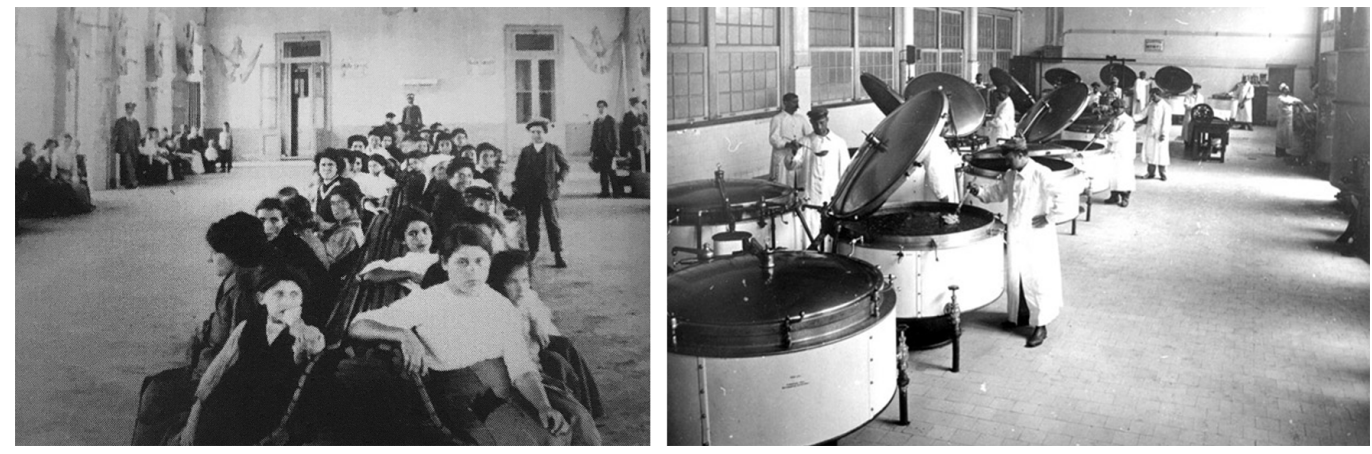

The building know-how was brought by the Italian community, numerically significant and, in an economic and social rise, supported also by the cultural integration policy adopted by the State. Among these, at first the Ley de Educación común in 1884 -which established a secular, universal and free schooling obligation for up to 14 years- which its 1899 implementation plan (the PPE-Plan de Edificación Escolar) provided the Capital and the whole national territory with a widespread network of buildings for primary school [2] [D'Amia, larossi 20I8], which design were made by the Italian Carlos Morra, with the support of Italian workers, technicians and designers.

Thanks to these integration policies, in Buenos Aires the migrates weren't concentrated in ethnically homogeneous neighbourhoods like Little Italy in New York, but they took up residence all over the city, with the only exception of La Boca, where there was a considerable community of Genoeses, hired like dockers, boatmen and pilots in the Riachuelo river port [3].The need of aggregation in a community, in which the common Italian origin was an em- 
bedded factor in a foreign land and in an urban dimension -already vast and more extensive in the eyes of migrants from the Italian provinces- from the mid-nineteenth century it gives the boot to the foundation of numerous mutual aid associations.

At first, they were organised on regional or scope basis (for example, to provide medical assistance on a solidarity basis), and clubs and societies became for the Italian-Argentine community centre of the associated life. After, thanks to the increase of the migratory fluxes, associations with other aims -politic, cultural, helpful, sports, recreational or educationalwere found, but also others referring to the location's barrio.

Some of these association had short life, others instead had a long one, thanks to the constant increase of their members, which allowed and made it necessary to have an own building intended for host them.

They had represented -and also nowadays- a fundamental point of reference for the Italian migrants spread through the metropolis, responding not only to the need of socialisation, but also to the satisfaction of primary needs, such as the health care or the professional education.

Most of the associations founded between the $X I X$ and the $X X$ centuries are nowadays present in the different barrios of the city and the buildings they occupied continue to interweave strong socio-cultural aggregation bounds into a demographically heterogenous and variable reality because of the still constant new migratory fluxes (for example, recently, from Venezuela).

It's a heritage which is often in a precarious state of conservation because of the weak protection policies in force in the Country and its condition of diffusion into the urban pattern. But it is certainly exemplar and worth of knowledge and valorisation, both as a tool of social and cultural integration and as an architectural example (fig. 2) (M.P.I.).
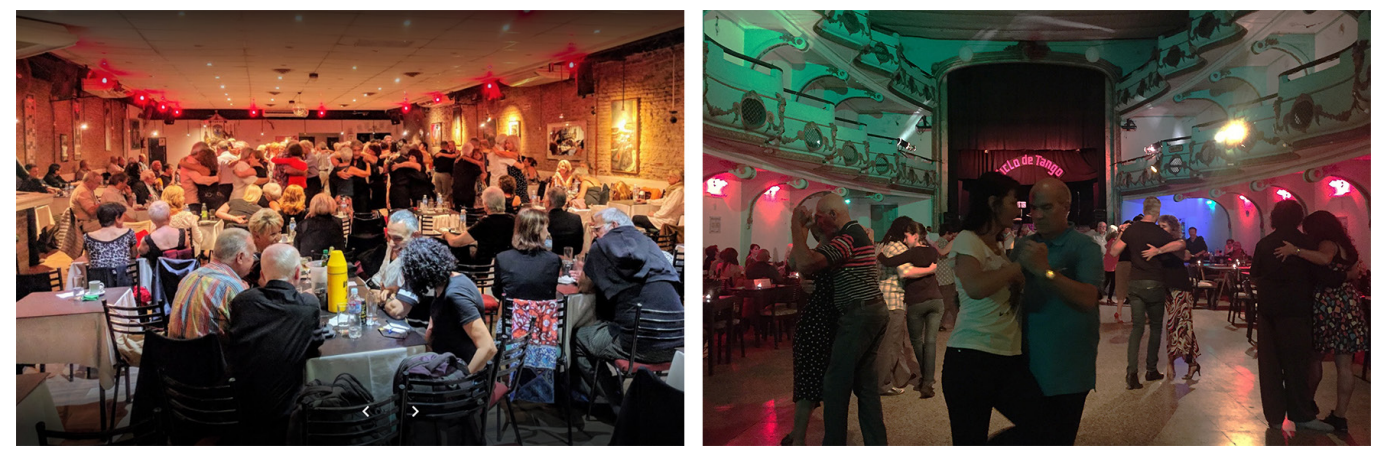

\section{Sources and survey methodologies}

The number of studies on the Italian migration phenomenon in Argentina and on the social and economic role made by the associationism [4] are very conspicuous [5].

Instead -if we exclude the most striking example with monumental value, such as the Unione e Benevolenza's building [6]- it seems that the totality of buildings used like a headquarters of the associations aren't much analysed, even though they are a conspicuous heritage with a considerable interest.

With the aim to understand the role of these buildings in the moment when Buenos Aires evolved itself into the Capital, the analysis was limited to the period between the 1880 and 1910, which corresponds to the moment of maximum intensity of the migratory fluxes. The work study begins comparing the first official records of foreign Italian associations, re- 
cognised by the Ministry of Foreign Affairs [7], to the list of those sill active in Buenos Aires, downloaded from the same Ministry website [8].

The cross-reference reading served to build a database comprising the geolocation of 91 buildings, these to provide a check in situ [9] of previously acquired data, a descriptive filing of those identified buildings still existing and a survey of case study, selected for their paradigmaticity (fig. 3).

The spread-out of the analysed heritage into the urban pattern, suggested to opt for a quickly survey methods, based on the integration of direct survey with photography and photogrammetry 3D, as well as documentary research, carried out in historical archive, where found, of each association.

In this way descriptive materials are gathered, and they represent the base for next researches and analysis about the comprehension for each building both of architectonical specific characters and of the relationship with the urban pattern and the structure of the whole city.

Referring to the analytic methodologies used, thanks to the spread of the analysed heritage and the specific aspect of the city of Buenos Aires, it seems that it was interesting examine in depth the architectural characters of the facades.

In Buenos Aires, indeed, the lack of restrictions, both on block's isotropic mesh building process (which originate from the extension of the XVI century founding grid, fixed by the Leyes de India) and on next centuries building reconstruction program [Gorelik, 1998], generated a various urban pattern under the typological and linguistic aspects. Thanks to this peculiarity, it seems to be fundamental carry out an in-depth analysis of the facades, because of the principal architectural aspect is in the tension between the cuadras' grid unlimited monotony and the buildings' facades semantic richness. (M.P.I.).

Fig. 3. On the left, extract of the database: in dark green the chosen cases study, in light green the buildings that still exist, in light yellow the ones that undergo change function, in red the demolished buildings. On the right, an example of the census sheet used (elaboration by the authors).

\begin{tabular}{|c|c|c|c|c|c|c|}
\hline Denominacion & Domililio & Data di i ondaraione & Telettono & Emal & Note & Dats \\
\hline 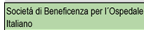 & Gascoson 450 , CABA & 1853 & 495844622 & & 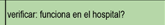 & 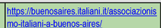 \\
\hline 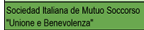 & 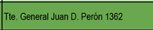 & 1814 19601858 & & & & 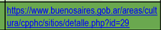 \\
\hline Socecti Nazinatab thature & 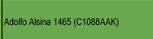 & 25 marсо 8381 & & 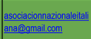 & 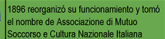 & 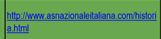 \\
\hline 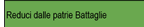 & 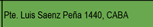 & Normbere 1869 & 43044476 & & 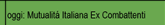 & \\
\hline Crevob atalemo & Leterd 1264 - C10125az & 1873 & & 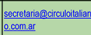 & 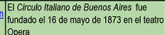 & 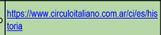 \\
\hline Uniner operai thatai & Samenib 1374 & 6 gemanao 1874 & & & 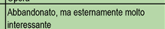 & 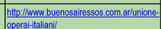 \\
\hline 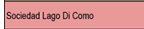 & 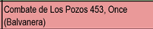 & 1 meran 1876 & 011 1942:24065 & & 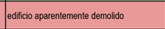 & \\
\hline Codoribiatarasa & Patara 55s & 8 spale 1877 & & & 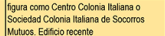 & \\
\hline Sistla dorlatia & Rvedtava 6 13 & 1978 & & & & \\
\hline Patra clavo & Cnne 1587 & 17 tebtrab 1878 & & & 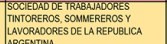 & \\
\hline Whia Unta & 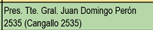 & 5 manocio 1378 & & & & \\
\hline Warpheria s Swoo & Carios Cavo 054 & $1800 m a 01879$ & & & & \\
\hline 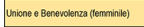 & Cevalos 778 & 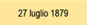 & & & & \\
\hline wing & 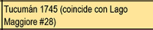 & 18 gemenein 1883 & & & 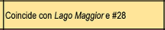 & \\
\hline Goinen latia & Boulcons sur Mer 730 & 22 laglolo 1883 & & & 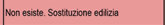 & \\
\hline Crenob Sannitico & & 8 Adicembre 1883 & & & & \\
\hline xx Sostentrone & Astan 2382 & 14 stentron 1984 & & & Edfricio dematito & \\
\hline Uninoror Mardideanle & sanzz Peñan 1046 & 5ercoron 1894 & & & & \\
\hline 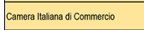 & 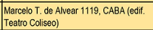 & 10centher 1884 & & & 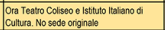 & \\
\hline Socielat Guchrie Cenererei & & 1884 & & & & \\
\hline Voghereses a i B Enenfferenza & 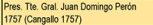 & S9umpo 1887 & & & & \\
\hline
\end{tabular}

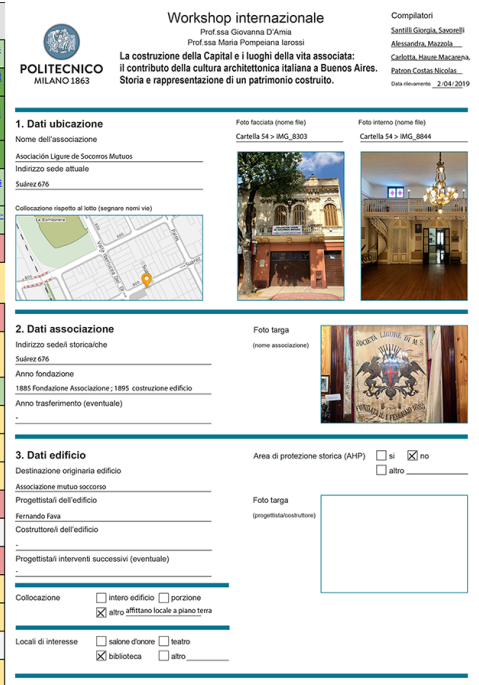

\section{Census, mapping, filing and survey of Italian associations' headquarters in Buenos Aires}

From the Ministry of Foreign Affairs' first census, result that in 1909 in the capital there was 75 active associations, instituted at the beginning of the mid- 19th century and among them there were 13 with several members that were higher than a thousand [10]. The following census published on Ministry of Foreign Affairs' website [I I] show as well that this number grow in time, as a result of closure or merger of some of these associations, just as the institution of new ones; so, in 2018, there was 9I Italian associations headquarters in the area of CABA-Comunidad Autónoma de Buenos Aires. 
Fig. 4. Existing and demolished associations buildings located into the districts of CABA (graphic elaboration by the authors).
Among these, from the survey in loco, 23 of them aren't found following the addresses contained in the ministerial reports; so it was possible to identify only 68 of them, among which $3 \mathrm{I}$ are still active and worthy of further in-depth study, as they have their own headquarter corresponding to an entire building, built with the purpose to accommodate them. So, this choice excluded from the analysis all cases of associations' headquarters that correspond to a building portion which haven't a recognisable independent architectural identity.

The so selected buildings were the object of additional survey, filling out for each of them a sheet with identified data both of the association and the building, relative state of preservation and use, name of the designer, description of architectural, typological, matter and stylistic aspects with relative photographic and graphic documentation compiled in loco. In the same time some associations' archive historic documents of interest for the purpose of the study were gathered, with especial attention on documents concerning building alterations during time.

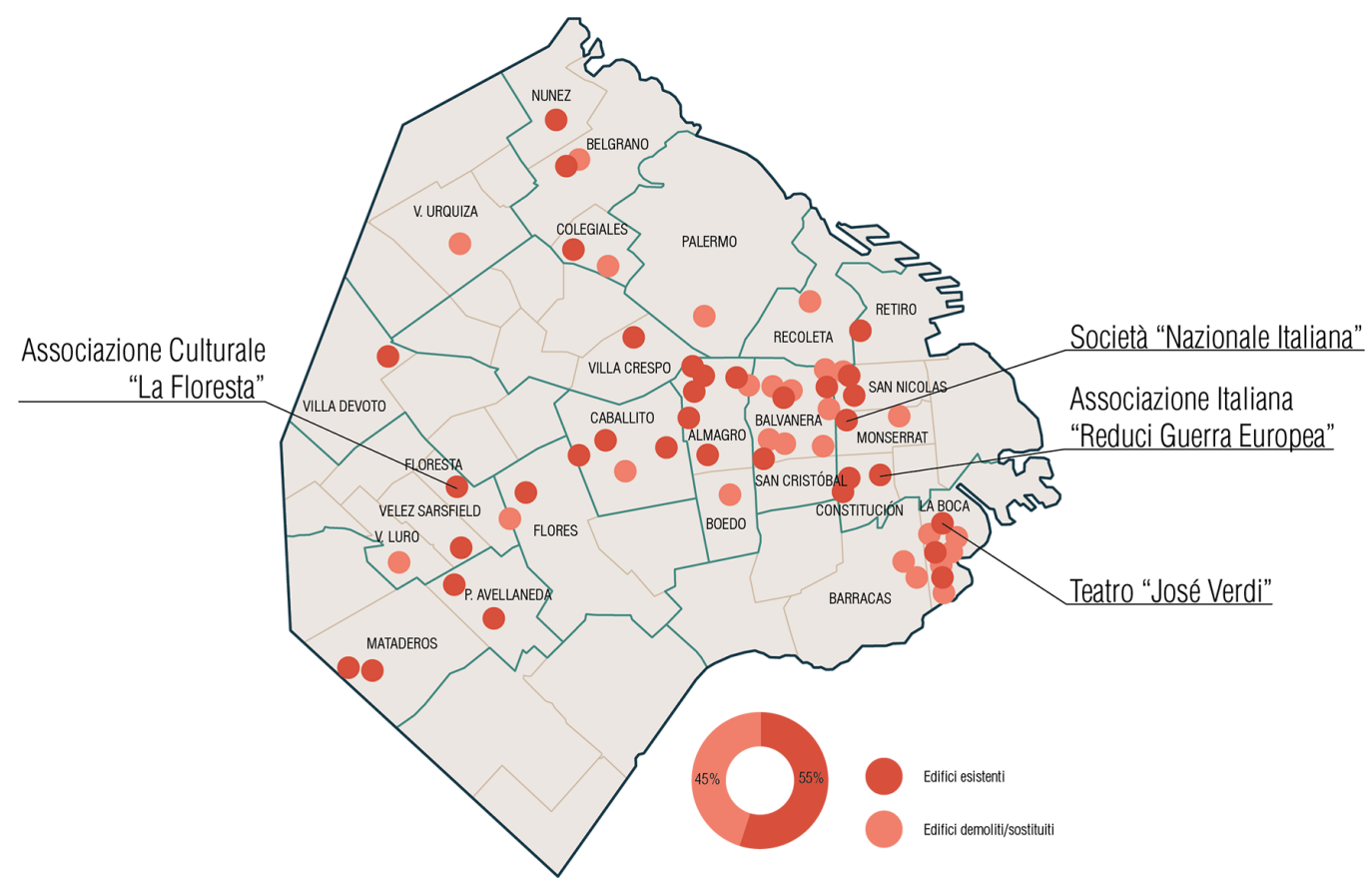

Based on that work and into consideration of the actual and easy accessibility to buildings, four cases study are selected:

- Teatro "José Verdi" (La Boca);

- Associazione Italiana "Reduci Guerra Europea" (Costitución);

- Associazione Culturale "La Floresta" (Floresta);

- Società "Nazionale Italiana" (Monserrat);

These buildings - selected in order to provide diversity about dating, author and location both into the city and into the block- were the object of a in-depth analysis, based on direct survey implemented by facades' and most significative inner rooms' digital models (such as halls and honour salons) [I2] (fig. 4). (M.P.I.). 


\section{Italian associations' buildings particular characters in Buenos Aires}

Referring to the census and mapping work done by computer, the spreading into the barrios of the associations is confirmed, finding a more concentration oh them in the barrios of Balvanera, La Boca and Almagro.

It has been also observed that they occupy a single parcel, developed in depth into the block and located in a barycentre position referring to the front of the cuadra even if there are some cases in which they are in the corner of it (fig. 5). the associations headquarter, in this way, are integral part of the curtain street made up by the continuous residential pattern.

Into the monotonous undiversified urban set and referring to the surrounding building pattern, the association's building is recognisable only for its architectural façade, used like a diaphragm between the public urban space and the inner one, as it want to visually communicate the role of socio-cultural root of its institution, denoted by the presence of a metal plaque or a sign, always well visible and with considerable dimensions (fig. 6).

The façade, in most of analysed cases, is symmetrical, divided by string course cornice and arrange into a hierarchy use of classic orders, ending with a crowning element composed of pediment or cornice with attic wall and sculptural elements (figs. 7, 8).

These facades have building elements not really irregular to the historic residential pattern's surrounding curtain wall, characterised by an altimetric development of one/two floors, laid on a raised base compared to the street quota.

Only some linguistic and decorative elements -such as the sign, the monumental crowing cornice and the presence of an opening in the centre of the façade, highlighted by a central balcony and equipped with a flag pole- allow to recognised them compared to the residential pattern, showing the common-house character for the community which is identified with them.

The inner distribution has the fulcrum, viewed as a prestige element, in the honour hall, sometimes double high developed, preceded by a vestibule equipped with a representation staircase (fig. 9).

In most cases the hall is like a theatre, equipped with one or two balcony and a proscenium with all its accessory rooms.

Some of these spaces are still used; they stage theatrical or musical performances and they are used most of the time like tango schools and milongas, dance halls, spread throughout the rioplatense area, earmark for tango.

Fig. 5. Cases study into their cuadras. An example of barycentric position (a:Theatre "José Verdi", b: Italian Association "Reduci Guerra Europea", c: Society "Nazionale Italiana") and of corner position (d: Cultural Association "La Floresta") (graphic elaboration by the authors).

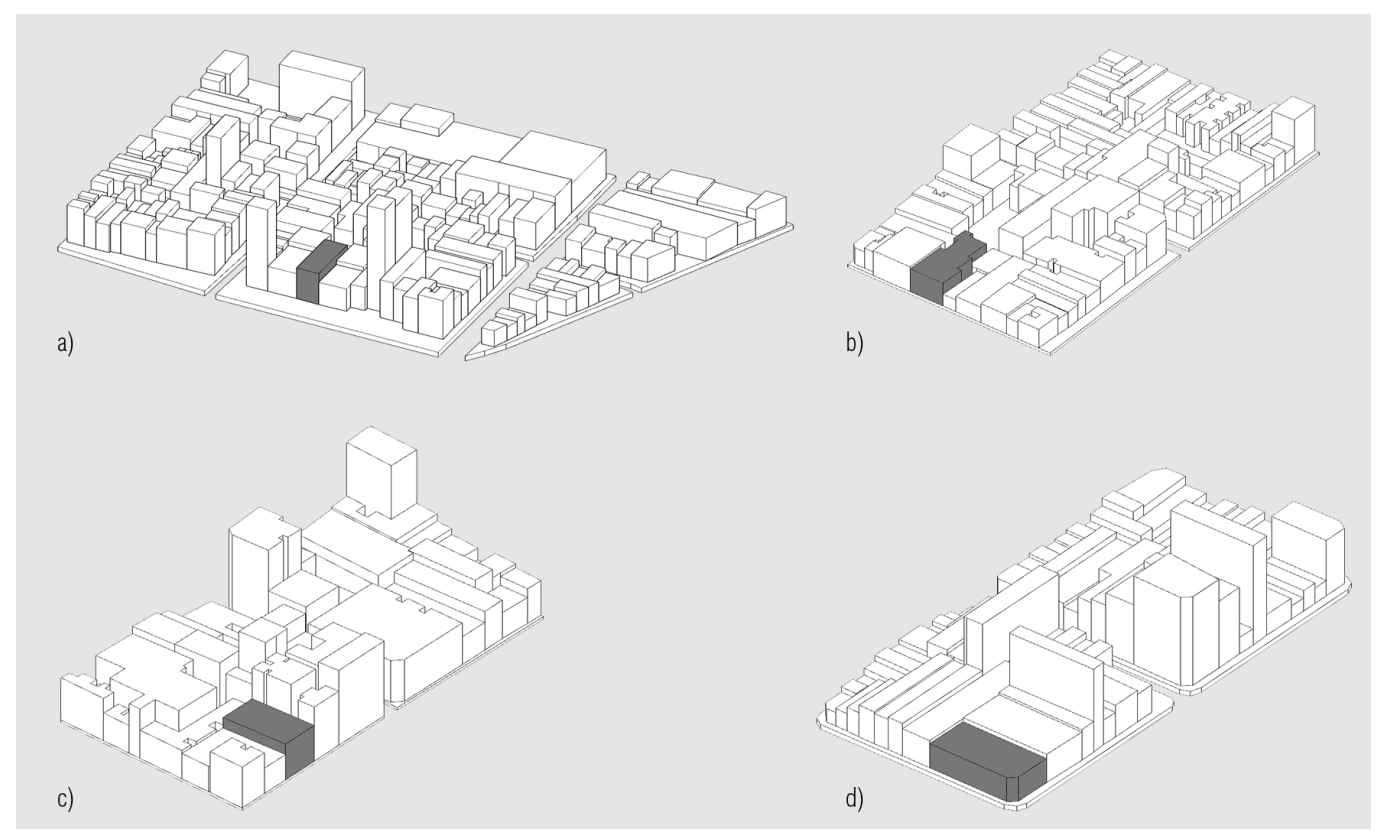


Fig. 6. Sings and plaques of some of the associations still active (photos by the authors).
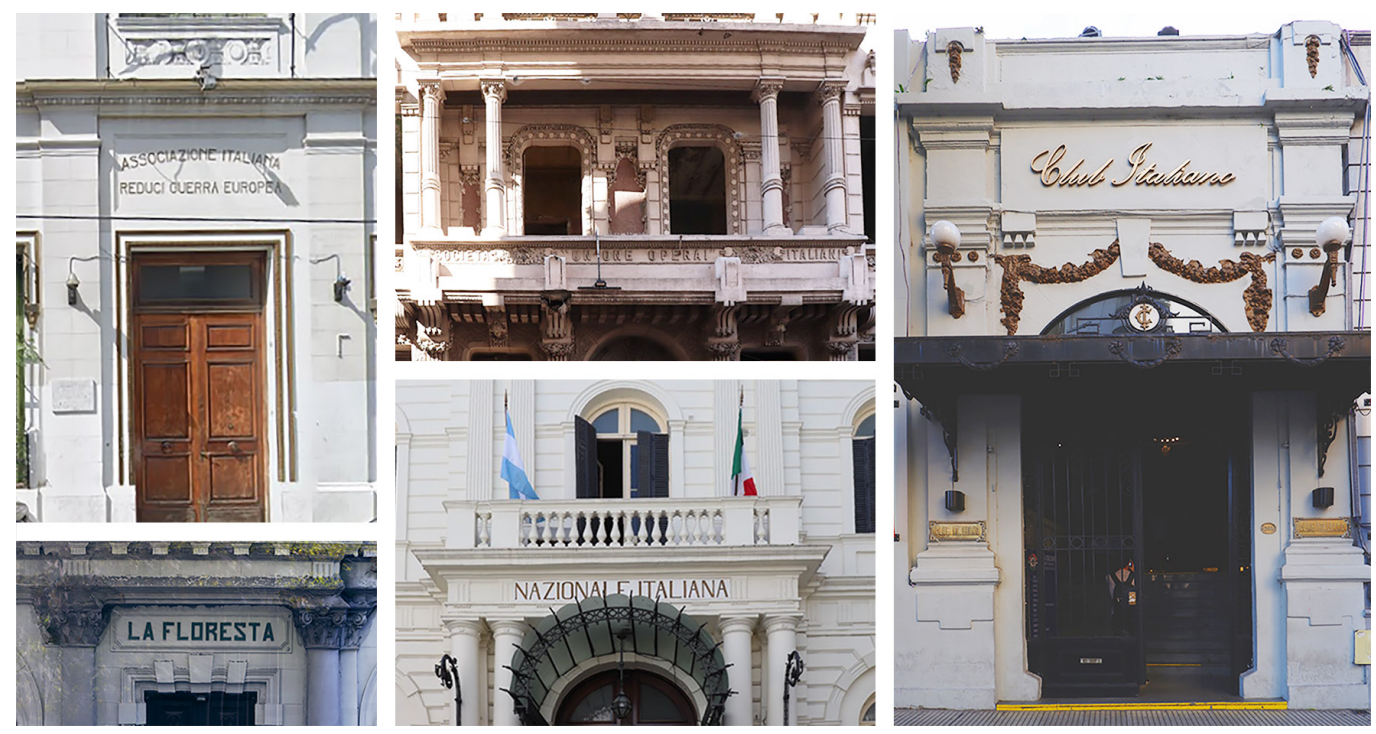

Fig. 7. Facades of Theatre "José Verdi" and Italian Association "Reduci

Guerra Europea" processed with Agisoft Photoscan (graphic elaboration by the authors)
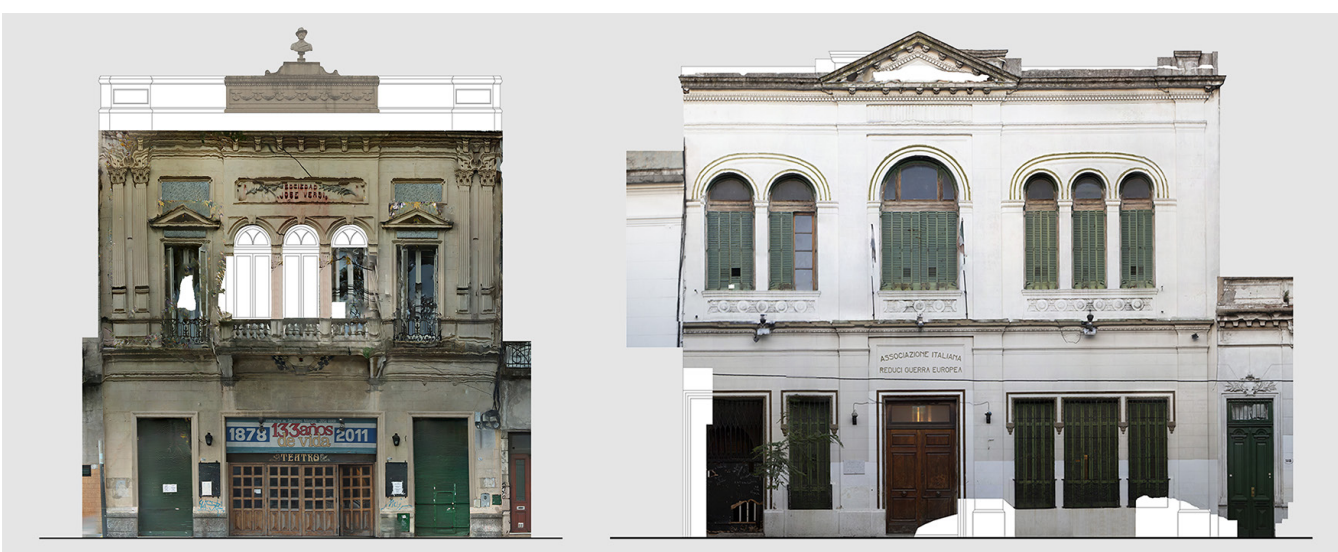

Fig. 8. Facades of Cultural Association "La Floresta" and Society "Nazionale

Italiana" processed

with Agisoft Photoscan

(graphic elaboration by the authors).

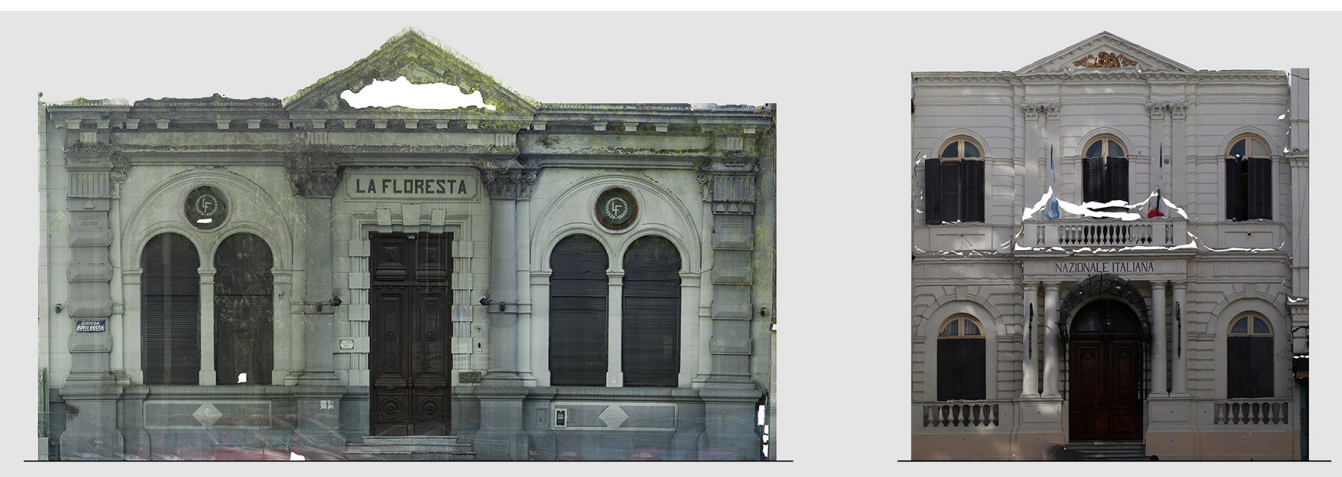


Of particular interest is the hall of Mutual Aid Society José Verdi, that is for all purposes a theatre (fig. I0). It is equipped with a proscenium, delimited by a scenic arch, opened to a large room, surrounded by two balconies and covered by a metal roof that can be opened. Unfortunately, the balconies are now condemned because they are unstable and the roof's opening mechanism isn't in function. However, the association is still represented not only a

Fig. 9. Picture and 3D process of the theatre room of Italian Association "José Verdi", (graphic elaboration by the authors)
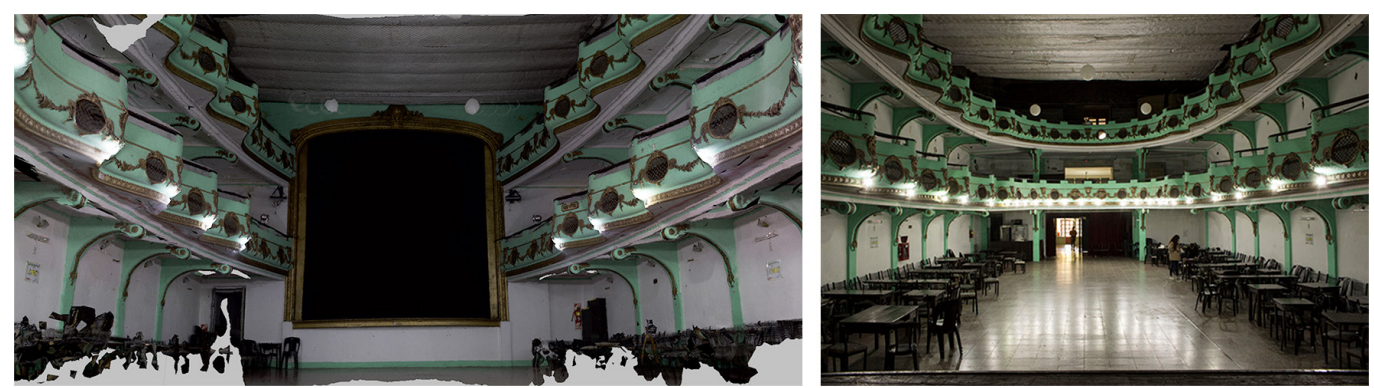

Fig. 10. Picture and 3D process of the theatre room of Theatre "José Verdi" (graphic elabor tion by the authors)
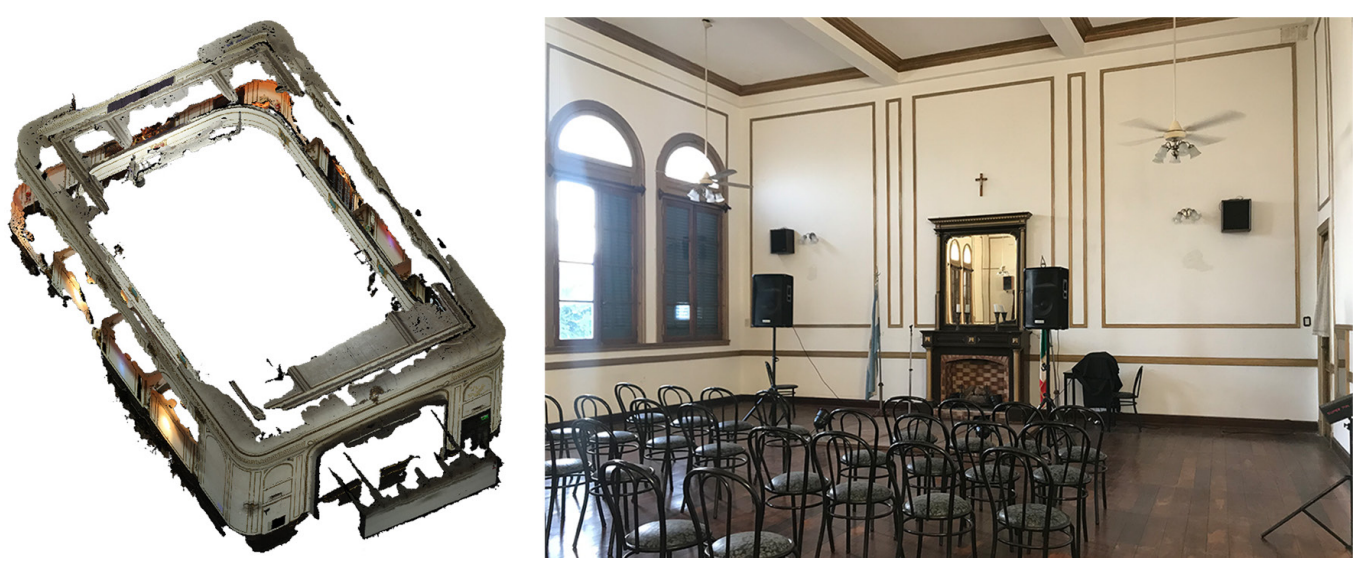

reference place for the residents of La Boca in which is located, but also an attractive pole for the original tango dancers [13] even if they come from other districts.

Around the hall and in upper stage there are service rooms, diversified depending on the activities and the service offered by each association. Indeed, there are offices, classrooms, libraries, meeting rooms, medical offices, little museum, bar and sports halls (fig. I I).

The analysis carried out on Italian associations in Buenos Aires showed that the inner distribution design, the position into the block and the city, connect the architecture of these buildings to the upper middle class domestic one's, meanwhile the façades composition and the presence of some decorative elements give to them a monumental connotation, affirming on the one hand their value as a home, and on the other their collective nature, as a reference for the social group that promotes their building and in which it recognises itself. (C.S.). 
Fig. I I. Arrangement of side rooms and the honour hall's service rooms for the associations "Reduci Guerra Europea" (a), "Nazionale Italiana"” (b) and the Theatre "lose Verdi" (c). (graphic elabo-

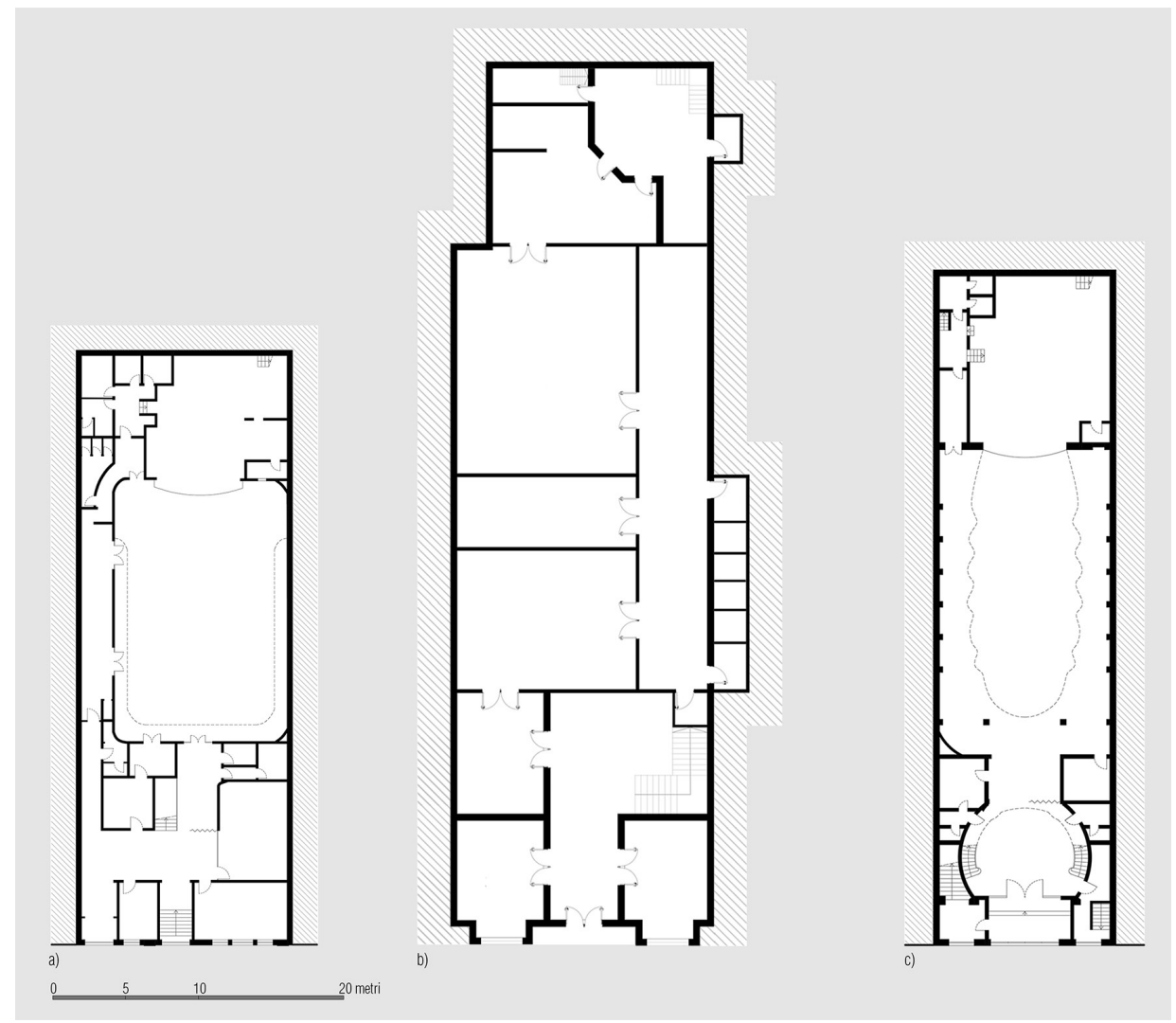

Notes

[I] The first Argentine national census (1869) observes that "the 59\% of all Italians in Argentina set up home in Buenos Aires.". In: Ministry of Foreign Affairs, 2003, p. 4.

[2] Brandariz 1998.

[3] Barzini 1902, pp. 19-2I; larossi 20 17, pp. I4-26.

[4] The binder role for the Italian-Argentine community made by the associationism was already recognised by L. Barzini since 1901, during his stay in Buenos Aires like a special reporter of Corriere della Sera: Barzini 1902, pp. I66-I72.

[5] For a historic reconstruction about the development of the associationism in Buenos Aires, see: Baily 1999 , pp. I74- I76.

[6] For a dissertation about the historic events of Unione e Benevolenza, the most ancient Italian association in Buenos Aires (founded in 1855), and also about a description of the vast and complex building which is its headquarters, in which there is a theatre, salons, offices, classrooms and meeting rooms, see: Cibotti 1986.

[7] Zuccarini 1909, pp.152-156.

[8] See: <http://www.esteri.it/mae/doc_osservatorio/gli_italiani_in_argentina.pdf>

[9] The census and survey operations were initialised into the workshop "Storia e Rappresentazione di un patrimonio costruito" conduced in April 2019 in Buenos Aires in partnership with Scuola AUIC-Architecttura, Urbanistica e Ingegneria delle Costruzioni of Politecnico di Milano and the Facultad de Arquitectura y urbanismo della Universidad de Belgrano a Buenos Aires in Buenos Aires. The data collected in situ were the object of an elaboration and an in-depth analysis carried in Italy.

[10] Zuccarini 1909, pp. I52-156.

[I I] See: <https://www.esteri.it/mae/doc_osservatorio/rapporto_italiani_argentina_logo.pdf>.

[12] Each 3D models were made with digital photomodelling, processing pictures captured with the camera CANON EOS IOOD into the software Agisoft Photoscan. In order to be effective for the purpose of the survey, these pictures are taken with a quickly modality using the same lens for all the set, paying attention that each frame is overlapped by at least $30 \%$ compared to the next one, in order to guarantee a correct coincidence of the homologous points.

[13] We have to remind that in 2009 the tango has been declared by the UNESCO Intangible Heritage of Humanity 


\section{References}

Baily Samuel L. (1999). Immigrants in the Lands of Promise. Italians in Buenos Aires and New York City, 1870-1914. Ithaca and London: Cornell University Press.

Barzini Luigi (1902). L'Argentina vista com'è. Milano:Tipografia del Corriere della Sera.

Brandariz Gustavo (1998). La arquitectura escolar de inspiración sarmentina. Buenos Aires: FADU-UBA.

Capocaccia Fabio, Pittarello Liliana, Rosso Del Brenna Giovanna (2016). Storie di emigrazione: architetti e costruttori italiani in America Latina. Genova: Stefano Termanini Editore.

Cibotti Ema (1986). Mutualismo y política en un estudio de caso. La sociedad 'Unione e Benevolenza' en Buenos Aires entre I 858 y | 865. In Devoto Fernando J. e Rosoli Gianfranco. L'Italia nella società argentina. Roma: Centro Studi Emigrazione, pp. $24 \mid-265$

Gutiérrez, Ramón (2004). Los italianos en la arquitectura argentina. Aproximaciones històricas. In Boletín CEDODAL: Italianos en la Arquitectura Argentina, gennaio.

larossi M. Pompeiana, Santacroce Cecilia (2019). The Schools as Heritage and a Tool for Political and Cultural Integration. The Buildings of the Plan de Edificación Escolar in Buenos Aires. In Della Torre Stefano, Bocciarelli Massimiliano, Daglio Laura, et al. Buildings for Education. Berlin: SpringerVerlag, pp. 73-85.

larossi M. Pompeiana (20 I 7). Laboratorio La Boca. Tracce d'Italia a Buenos Aires. Firenze: Altralinea Edizioni.

Zuccarini Emilio (1909). Il lavoro degli Italiani nella Repubblica argentina: dal 15 I 6 al 19 I0: studi, leggende e ricerche. Buenos Aires: Oficine gráfiche de la Compañia General de Fósforos.

Ministero degli Affari Esteri. Osservatorio sul Lavoro e sulla Formazione degli Italiani all'Estero. Gli Italiani in Argentina. In: <http:// www.esteri.it/mae/doc_osservatorio/gli_italiani_in_argentina.pdf $>$; $<$ https://www.esteri.it/mae/doc_osservatorio/rapporto_ italiani_argentina_logo.pdf>

Portal de la Comunidad Italiana en Argentina <http://www.feditalia.org.ar/>.

\section{Authors}

Maria Pompeiana larossi, Politecnico di Milano, mariapompeiana.iarossi@polimi.it

Cecilia Santacroce, Politecnico di Milano, cecilia.santacroce@polimi.it

To cite this chapter. larossi Maria Pompeiana, Santacroce Cecilia (2020). II legato dei legami. Le sedi storiche dell'associazionismo italiano a Buenos Aires/Legacy of links. The historical headquarters of Italian associationism in Buenos Aires. In Arena A., Arena M., Brandolino R.G., Colistra D. Ginex G., Mediati D., Nucifora S., Raffa P. (a cura di). Connettere. Un disegno per annodare e tessere. Atti del $42^{\circ}$ Convegno Internazionale dei Docent delle Discipline della Rappresentazione/Connecting. Drawing for weaving relationships. Proceedings of the 42th International Conference of Representation Disciplines Teachers. Milano: FrancoAngeli, pp. 2292-231।. 